\title{
THE GROTHENDIECK CONSTANT IS STRICTLY SMALLER THAN KRIVINE'S BOUND
}

\author{
MARK BRAVERMAN ${ }^{1}$, KONSTANTIN MAKARYCHEV $^{2}$, \\ YURY MAKARYCHEV ${ }^{3}$ and ASSAF NAOR ${ }^{4}$ \\ ${ }^{1}$ Princeton University, Department of Computer Science, 35 Olden Street, Princeton, NJ 08540, \\ USA; email: mbraverm@cs.princeton.edu \\ ${ }^{2}$ Microsoft Research, One Microsoft Way, Redmond, WA 98052, USA; \\ email: komakary@microsoft.com \\ ${ }^{3}$ Toyota Technological Institute at Chicago, 6045 S. Kenwood Ave., Chicago, IL 60637, USA; \\ email: yury@ttic.edu \\ ${ }^{4}$ New York University, Courant Institute, 251 Mercer Street, New York, NY 10012, USA; \\ email: naor@cims.nyu.edu
}

Received 26 August 2013; accepted 21 October 2013

\begin{abstract}
The (real) Grothendieck constant $K_{G}$ is the infimum over those $K \in(0, \infty)$ such that for every $m, n \in \mathbb{N}$ and every $m \times n$ real matrix $\left(a_{i j}\right)$ we have

$$
\max _{\left\{x_{i}\right\}_{i=1}^{m},\left\{y_{j}\right\}_{j=1}^{n} \subseteq S^{n+m-1}} \sum_{i=1}^{m} \sum_{j=1}^{n} a_{i j}\left\langle x_{i}, y_{j}\right\rangle \leqslant K \max _{\left\{\varepsilon_{i}\right\}_{i=1}^{m},\left\{\delta_{j}\right\}_{j=1}^{n} \subseteq\{-1,1\}} \sum_{i=1}^{m} \sum_{j=1}^{n} a_{i j} \varepsilon_{i} \delta_{j} .
$$

The classical Grothendieck inequality asserts the nonobvious fact that the above inequality does hold true for some $K \in(0, \infty)$ that is independent of $m, n$ and $\left(a_{i j}\right)$. Since Grothendieck's 1953 discovery of this powerful theorem, it has found numerous applications in a variety of areas, but, despite attracting a lot of attention, the exact value of the Grothendieck constant $K_{G}$ remains a mystery. The last progress on this problem was in 1977 , when Krivine proved that $K_{G} \leqslant \pi / 2 \log (1+\sqrt{2})$ and conjectured that his bound is optimal. Krivine's conjecture has been restated repeatedly since 1977 , resulting in focusing the subsequent research on the search for examples of matrices $\left(a_{i j}\right)$ which exhibit (asymptotically, as $\left.m, n \rightarrow \infty\right)$ a lower bound on $K_{G}$ that matches Krivine's bound. Here, we obtain an improved Grothendieck inequality that holds for all matrices $\left(a_{i j}\right)$ and yields a bound $K_{G}<\pi / 2 \log (1+\sqrt{2})-\varepsilon_{0}$ for some effective constant $\varepsilon_{0}>0$. Other than disproving Krivine's conjecture, and along the way also disproving an intermediate conjecture of König that was made in 2000 as a step towards Krivine's conjecture, our main contribution is conceptual: despite dealing with a binary rounding problem, random
\end{abstract}


two-dimensional projections, when combined with a careful partition of $\mathbb{R}^{2}$ in order to round the projected vectors to values in $\{-1,1\}$, perform better than the ubiquitous random hyperplane technique. By establishing the usefulness of higher-dimensional rounding schemes, this fact has consequences in approximation algorithms. Specifically, it yields the best known polynomial-time approximation algorithm for the Frieze-Kannan Cut Norm problem, a generic and well-studied optimization problem with many applications.

2010 Mathematics Subject Classification: 15A60 (primary); 15A45, 90C27, 90C22 (secondary)

\section{Overview}

In 1953, Grothendieck proved a theorem that has the following matrix-theoretic formulation: there exists a universal constant $K \in[1, \infty)$ such that, given an $m$ by $n$ matrix $A=\left(a_{i j}\right)$ with real entries and $m+n$ unit vectors $x_{1}, \ldots, x_{m}, y_{1}, \ldots, y_{n}$ in Hilbert space, one can find signs $\varepsilon_{1}, \ldots, \varepsilon_{m}, \delta_{1}, \ldots, \delta_{n} \in\{-1,1\}$ for which $\sum_{i=1}^{m} \sum_{j=1}^{n} a_{i j}\left\langle x_{i}, y_{j}\right\rangle \leqslant K \sum_{i=1}^{m} \sum_{j=1}^{n} a_{i j} \varepsilon_{i} \delta_{j}$. The key point here is that $K$ is independent of $m$ and $n$, the matrix $A$, and the unit vectors $x_{1}, \ldots, x_{m}$ and $y_{1}, \ldots, y_{n}$. The infimum over those $K$ for which the above statement holds true is called the Grothendieck constant, and is denoted $K_{G}$. This innocent-looking inequality had profound impact on a variety of disciplines, ranging from its roots in functional analysis to harmonic analysis, operator theory, quantum mechanics, and computer science. The number $K_{G}$ has equivalent formulations as a fundamental constant in convex geometry, quantum information theory, and complexity theory, but, despite major efforts by many researchers over the past 60 years, its exact value (indeed, even its second digit) remains unknown. While it would be interesting to know the first few digits of $K_{G}$, or perhaps to relate it to other classical constants, the search for the value of $K_{G}$ is mainly a way to encode the fundamental question of understanding the structure of the matrices $A$ and point configurations $x_{1}, \ldots, x_{m}, y_{1}, \ldots, y_{n}$ that are asymptotically 'most difficult' for Grothendieck's inequality. In 1977, Krivine proved that $K_{G}$ is at most $\pi /(2 \log (1+\sqrt{2}))$, and conjectured that $K_{G}$ actually equals this number, a conjecture that was based on an intuitive expectation that a certain specific family of matrices $A$ saturates Grothendieck's inequality. Krivine's conjecture became the central open problem in this area, a problem that we resolve negatively by obtaining the first improved upper bound on $K_{G}$ since 1977 . Rather than analyzing a specific example of matrices, as required by an affirmative solution of Krivine's conjecture, here we find a new method that performs better than Krivine's method on all matrices. After applying a certain explicit deterministic transformation of the input vectors $x_{1}, \ldots, x_{m}, y_{1}, \ldots, y_{n}$, we proceed to project them onto a random two-dimensional plane. By carefully partitioning the plane into two regions, one can associate a \pm 1 sign to each of the vectors based on the part of the planar partition in which their random projection landed.

\section{Introduction}

In his 1953 Résumé [14], Grothendieck proved a theorem that he called 'le théorème fondamental de la théorie metrique des produits tensoriels'. This result is known today as Grothendieck's inequality. An equivalent formulation of Grothendieck's inequality, due to Lindenstrauss and Pełczyński [26], states that there exists a universal constant $K \in(0, \infty)$ such that for every $m, n \in \mathbb{N}$, every $m \times n$ matrix $\left(a_{i j}\right)$ with real entries, and every $m+n$ unit vectors 
$x_{1}, \ldots, x_{m}, y_{1}, \ldots, y_{n} \in S^{m+n-1}$, there exist $\varepsilon_{1}, \ldots, \varepsilon_{m}, \delta_{1}, \ldots, \delta_{n} \in\{-1,1\}$ satisfying

$$
\sum_{i=1}^{m} \sum_{j=1}^{n} a_{i j}\left\langle x_{i}, y_{j}\right\rangle \leqslant K \sum_{i=1}^{m} \sum_{j=1}^{n} a_{i j} \varepsilon_{i} \delta_{j} .
$$

Here, $\langle\cdot, \cdot\rangle$ denotes the standard scalar product on $\mathbb{R}^{m+n}$. The infimum over those $K \in(0, \infty)$ for which (1.1) holds true is called the Grothendieck constant, and is denoted $K_{G}$.

Grothendieck's inequality is important to several disciplines, including the geometry of Banach spaces, $C^{*}$ algebras, harmonic analysis, operator spaces, quantum mechanics, and computer science. Rather than attempting to explain the ramifications of Grothendieck's inequality, we refer to the books [1,5,9, $10,13,17,27,32,37$ ] and especially Pisier's recent survey [33]. The survey [21] is devoted to Grothendieck's inequality in computer science; Section 2 below contains a brief discussion of this topic.

Problem 3 of Grothendieck's Résumé asks for the determination of the exact value of $K_{G}$. This problem remains open despite major effort by many mathematicians. In fact, even though $K_{G}$ occurs in numerous mathematical theorems, and has equivalent interpretations as a key quantity in physics $[11,38]$ and computer science [2,34], we currently do not even know what the second digit of $K_{G}$ is; the best known bounds [23,35] are $K_{G} \in(1.676,1.782)$.

Following the upper bounds on $K_{G}$ obtained in [14,26,36] (see also the alternative proofs of (1.1) in [6,10,18, 28, 29,31], yielding worse bounds on $K_{G}$ ), progress on Grothendieck's Problem 3 halted after a beautiful 1977 theorem of Krivine [23], who proved that

$$
K_{G} \leqslant \frac{\pi}{2 \log (1+\sqrt{2})}(=1.782 \ldots) .
$$

One reason for this lack of improvement since 1977 is that Krivine conjectured [23] that his bound is actually the exact value of $K_{G}$. Krivine's conjecture has become the leading conjecture in this area, and as such it has been restated repeatedly in subsequent publications; see for example [7, 22, 24, 31,32]. Quoting Pisier's book [32, p. 64], 'The best known estimate for $K_{G}$ is due to Krivine, who proved (see [Kri3]) that $K_{G} \leqslant 2 / \log (1+\sqrt{2})=1.782 \ldots$ and conjectured that this is the exact value of $K_{G} \ldots$ Krivine claims that he checked $K_{G}>\pi / 2$, and he has convincing (unpublished) evidence that his bound is sharp.' The belief that the estimate (1.2) is the exact value of $K_{G}$ focused research on finding examples of matrices $\left(a_{i j}\right)$ that exhibit a matching lower bound on $K_{G}$. Following work of Haagerup, Tomczak-Jaegermann, and König, the search for such matrices led in 2000 to a clean intermediate conjecture of 
König [22], on maximizers of a certain oscillatory integral operator, that was shown to imply Krivine's conjecture; we will explain this conjecture, which we resolve in this paper, in Section 1.1. Here, we prove that Krivine's conjecture is false, thus obtaining the best known upper bound on $K_{G}$.

THEOREM 1.1. There exists $\varepsilon_{0}>0$ such that

$$
K_{G}<\frac{\pi}{2 \log (1+\sqrt{2})}-\varepsilon_{0} .
$$

We stress that our proof is effective, and it readily yields a concrete positive lower bound on $\varepsilon_{0}$. We chose not to state an explicit new upper bound on the Grothendieck constant since we know that our estimate is suboptimal: see Remark 3.3. Section 3 contains a discussion of potential improvements of our bound, based on challenging open problems that conceivably might even lead to an exact evaluation of $K_{G}$.

REMARK 1.2. There has also been major effort to estimate the complex Grothendieck constant $[8,14,31]$; the best known upper bound in this case is due to Haagerup [16]. We did not investigate this issue here, partly because for complex scalars there is no clean conjectured exact value of the Grothendieck constant in the spirit of Krivine's conjecture. Nevertheless, it is conceivable that our approach can improve Haagerup's bound on the complex Grothendieck constant as well. We leave this research direction open for future investigations.

In our opinion, the interest in the exact value of $K_{G}$ does not necessarily arise from the importance of this constant itself, though the reinterpretation of $K_{G}$ as a fundamental constant in physics and computer science makes it even more interesting to know at least its first few digits. Rather, we believe that it is very interesting to understand the geometric configuration of the unit vectors $x_{1}, \ldots, x_{m}, y_{1}, \ldots, y_{n} \in S^{m+n-1}$ (and matrix $a_{i j}$ ) which makes the inequality (1.1) 'most difficult'. This issue is related to the 'rounding problem' in theoretical computer science; see Section 2. With this in mind, Krivine's conjecture corresponds to a natural geometric intuition about the worst spherical configuration for Grothendieck's inequality. This geometric picture has been crystallized and cleanly formulated as an extremal analytic/geometric problem due to the works of Haagerup, König, and Tomczak-Jaegermann. We shall now explain this issue, since one of the main conceptual consequences of Theorem 1.1 is that the geometric picture behind Grothendieck's inequality that was previously believed to be true is actually false. Along the way, we resolve a conjecture of König [22].

1.1. König's problem. One can reformulate Grothendieck's inequality using integral operators (see [22]). Given a measure space $(\Omega, \mu)$ and a kernel 
$K \in L_{1}(\Omega \times \Omega, \mu \times \mu)$, consider the integral operator $T_{K}: L_{\infty}(\Omega, \mu) \rightarrow L_{1}(\Omega, \mu)$ induced by $K$, that is,

$$
T_{K} f(x) \stackrel{\text { def }}{=} \int_{\Omega} f(y) K(x, y) d \mu(y) .
$$

Grothendieck's inequality asserts that for every $f, g \in L_{\infty}\left(\Omega, \mu ; \ell_{2}\right)$, that is, two bounded measurable functions with values in Hilbert space,

$$
\begin{aligned}
& \int_{\Omega} \int_{\Omega} K(x, y)\langle f(x), g(y)\rangle d \mu(x) d \mu(y) \\
& \quad \leqslant K_{G}\left\|T_{K}\right\|_{L_{\infty}(\Omega, \mu) \rightarrow L_{1}(\Omega, \mu)}\|g\|_{L_{\infty}\left(\Omega, \mu ; \ell_{2}\right)}\|f\|_{L_{\infty}\left(\Omega, \mu ; \ell_{2}\right)} .
\end{aligned}
$$

König [22], citing unpublished computations of Haagerup, asserts that the assumption $K_{G}=\pi /(2 \log (1+\sqrt{2}))$ suggests that the oscillatory Gaussian kernel $K: \mathbb{R}^{n} \times \mathbb{R}^{n} \rightarrow \mathbb{R}$ given by

$$
K(x, y) \stackrel{\text { def }}{=} \exp \left(-\frac{\|x\|_{2}^{2}+\|y\|_{2}^{2}}{2}\right) \sin (\langle x, y\rangle)
$$

should be extremal for Grothendieck's inequality in the asymptotic sense, that is, for $n \rightarrow \infty$. In the rest of this paper, $K$ will always stand for the kernel appearing in (1.4), and the corresponding bilinear form $B_{K}: L_{\infty}\left(\mathbb{R}^{n}\right) \times L_{\infty}\left(\mathbb{R}^{n}\right) \rightarrow \mathbb{R}$ will be given by

$$
B_{K}(f, g) \stackrel{\text { def }}{=} \int_{\mathbb{R}^{n}} \int_{\mathbb{R}^{n}} f(x) g(y) K(x, y) d x d y .
$$

The above discussion led König to make the following conjecture.

Conjecture 1.3 (König [22]). Define $f_{0}: \mathbb{R}^{n} \rightarrow\{-1,1\}$ by $f_{0}\left(x_{1}, \ldots, x_{n}\right)=$ $\operatorname{sign}\left(x_{1}\right)$. Then $B_{K}(f, g) \leqslant B_{K}\left(f_{0}, f_{0}\right)$ for every $n \in \mathbb{N}$ and every measurable $f, g: \mathbb{R}^{n} \rightarrow\{-1,1\}$.

In [22], the following result of König and Tomczak-Jaegermann is proved.

PROPOSITION 1.2 (König and Tomczak-Jaegermann [22]). A positive answer to Conjecture 1.3 would imply that $K_{G}=\frac{\pi}{2 \log (1+\sqrt{2})}$.

Proposition 1.2 itself can be viewed as motivation for Conjecture 1.3, since it is consistent with Haagerup's work and Krivine's conjecture. But, there are additional reasons why Conjecture 1.3 is natural. First of all, we know due to Lieb's work [25] that general Gaussian kernels, when viewed as operators from $L_{p}\left(\mathbb{R}^{n}\right)$ to $L_{q}\left(\mathbb{R}^{n}\right)$, have only Gaussian maximizers provided $p$ and $q$ satisfy certain conditions. The kernel $K$ does not fit into Lieb's framework, since it is the imaginary part of a Gaussian kernel (the Gaussian Fourier transform) rather than an actual Gaussian kernel, and moreover the range $p=\infty$ and $q=1$ is 
not covered by Lieb's theorem. Nevertheless, in light of Lieb's theorem, one might expect that maximizers of kernels of this type have a simple structure, which could be viewed as a weak justification of Conjecture 1.3. A much more substantial justification of Conjecture 1.3 is that in [22] König announced an unpublished result that he obtained jointly with Tomczak-Jaegermann, asserting that Conjecture 1.3 is true for $n=1$.

THEOREM 1.4. For every Lebesgue measurable $f, g: \mathbb{R} \rightarrow\{-1,1\}$, we have

$$
\begin{aligned}
& \int_{\mathbb{R}} \int_{\mathbb{R}} f(x) g(y) \exp \left(-\frac{x^{2}+y^{2}}{2}\right) \sin (x y) d x d y \\
& \quad \leqslant \int_{\mathbb{R}} \int_{\mathbb{R}} \operatorname{sign}(x) \operatorname{sign}(y) \exp \left(-\frac{x^{2}+y^{2}}{2}\right) \sin (x y) d x d y \\
& \quad=2 \sqrt{2} \log (1+\sqrt{2}) .
\end{aligned}
$$

Moreover, equality in (1.6) is attained only when $f(x)=g(x)=\operatorname{sign}(x)$ almost everywhere or $f(x)=g(x)=-\operatorname{sign}(x)$ almost everywhere.

We believe that it is important to have a published proof of Theorem 1.4, and for this reason we prove it in Section 6. Conceivably, our proof is similar to the unpublished proof of König and Tomczak-Jaegermann, though they might have found a different explanation of this phenomenon. Since Theorem 1.1 combined with Proposition 1.2 implies that König's conjecture is false, and as we shall see it is false already for $n=2$, Theorem 1.4 highlights special behavior of the one-dimensional case.

Our proof of Theorem 1.1 starts by disproving König's conjecture for $n=2$. This is done in Section 4. Obtaining an improved upper bound on the Grothendieck constant requires a substantial amount of additional work that uses the counterexample to Conjecture 1.3. This is carried out in Section 5. The failure of König's conjecture shows that the situation is more complicated than originally hoped, and in particular that for $n>1$ the maximizers of the kernel $K$ have a truly high-dimensional behavior. This more complicated geometric picture highlights the availability of high-dimensional rounding schemes that are more sophisticated (and better) than 'hyperplane rounding'. These issues are discussed in Sections 2 and 3.

\section{Krivine-type rounding schemes and algorithmic implications}

Consider the following optimization problem. Given an $m \times n$ matrix $A=\left(a_{i j}\right)$, compute in polynomial time the value

$$
\mathrm{OPT}(A) \stackrel{\text { def }}{=} \max _{\varepsilon_{1}, \ldots, \varepsilon_{m}, \delta_{1}, \ldots, \delta_{n} \in\{-1,1\}} \sum_{i=1}^{m} \sum_{j=1}^{n} a_{i j} \varepsilon_{i} \delta_{j} .
$$


We refer to $[2,21]$ for a discussion of the combinatorial significance of this problem. It suffices to say here that it relates to the problem of computing efficiently the Cut Norm of a matrix, which is a subroutine in a variety of applications, starting with the pioneering work of Frieze and Kannan [12]. Special choices of matrices $A$ in (2.1) lead to specific problems of interest, including efficient construction of Szemerédi partitions [2].

As shown in [2], there exists $\delta_{0}>0$ such that the existence of a polynomial-time algorithm that outputs a number that is guaranteed to be within a factor of $1+\delta_{0}$ of $\mathrm{OPT}(A)$ would imply that $\mathrm{P}=\mathrm{NP}$. But, since the quantity

$$
\operatorname{SDP}(A) \stackrel{\text { def }}{=} \max _{x_{1}, \ldots, x_{m}, y_{1}, \ldots, y_{n} \in S^{m+n-1}} \sum_{i=1}^{m} \sum_{j=1}^{m} a_{i j}\left\langle x_{i}, y_{j}\right\rangle
$$

can be computed in polynomial time with arbitrarily good precision (it is a semidefinite program [15]), Grothendieck's inequality tells us that the polynomial-time algorithm that outputs the number $\operatorname{SDP}(A)$ is always within a factor of $K_{G}$ of OPT $(A)$.

Remarkably, the work of Raghavendra and Steurer [34] shows that $K_{G}$ has a complexity-theoretic interpretation: it is likely that no polynomial-time algorithm can approximate $\mathrm{OPT}(A)$ to within a factor smaller than $K_{G}$. More precisely, it is shown in [34] that $K_{G}$ is the Unique Games hardness threshold of the problem of computing OPT $(A)$. To explain what this means, we briefly recall Khot's Unique Games conjecture [19] (the version described below is equivalent to the original one, as shown in [20]).

Khot's conjecture asserts that for every $\varepsilon>0$ there exists a prime $p=p(\varepsilon) \in \mathbb{N}$ such that there is no polynomial-time algorithm that, given $n \in \mathbb{N}$ and a system of $m$ linear equations in $n$ variables of the form

$$
x_{i}-x_{j} \equiv c_{i j}(\bmod p)
$$

for some $c_{i j} \in \mathbb{N}$, determines whether there exists an assignment of an integer value to each variable $x_{i}$ such that at least $(1-\varepsilon) m$ of the equations are satisfied, or whether no assignment of such values can satisfy more than $\varepsilon m$ of the equations (if neither of these possibilities occurs, then an arbitrary output is allowed).

The Unique Games conjecture is by now a common assumption that has numerous applications in computational complexity. We have already seen that there exists a polynomial-time algorithm that computes $\mathrm{OPT}(A)$ to within a factor of $K_{G}$. The Raghavendra-Steurer theorem says that, if there were a polynomial-time algorithm $A L G$ that computes $\mathrm{OPT}(A)$ to within a factor $c<$ $K_{G}$, then the Unique Games conjecture would be false. This means that there is $\varepsilon=\varepsilon_{c} \in(0,1)$ such that, for all primes $p$, Raghavendra and Steurer design an algorithm that makes one call to the algorithm $A L G$, with at most polynomially many additional Turing machine steps, which successfully solves the problem 
described above on the satisfiability of linear equations modulo $p$. Note that Raghavendra and Steurer manage to do this despite the fact that the value of $K_{G}$ is unknown.

Theorem 1.1 yields the first improved upper bound on the Unique Games hardness threshold of the OPT $(A)$ computation problem since Krivine's 1977 bound. As we shall see, what hides behind Theorem 1.1 is also a new algorithmic method which is of independent interest. To explain this, note that the above discussion dealt with the problem of computing the number $\mathrm{OPT}(A)$. But it is actually of greater interest to find in polynomial time signs $\varepsilon_{1}, \ldots, \varepsilon_{m}, \delta_{1}, \ldots, \delta_{n} \in\{-1,1\}$, from among all such $2^{m+n}$ choices of signs, for which $\sum_{i=1}^{m} \sum_{j=1}^{n} a_{i j} \varepsilon_{i} \delta_{j}$ is at least a constant multiple $\mathrm{OPT}(A)$. This amounts to a 'rounding problem': we need to find a procedure that, given vectors $x_{1}, \ldots, x_{m}, y_{1}, \ldots, y_{n} \in S^{m+n-1}$, produces signs $\varepsilon_{1}, \ldots, \varepsilon_{m}, \delta_{1}, \ldots, \delta_{n} \in\{-1,1\}$ whose existence is ensured by Grothendieck's inequality (1.1).

Krivine's proof of (1.2) is based on a clever two-step rounding procedure. We shall now describe a generalization of Krivine's method.

DeFinition 2.1 (Krivine rounding scheme). Fix $k \in \mathbb{N}$, and assume that we are given two odd measurable functions $f, g: \mathbb{R}^{k} \rightarrow\{-1,1\}$. Let $G_{1}, G_{2} \in \mathbb{R}^{k}$ be independent random vectors that are distributed according to the standard Gaussian measure on $\mathbb{R}^{k}$, that is, the measure with density $x \mapsto e^{-\|x\|_{2}^{2} / 2} /(2 \pi)^{k / 2}$. For $t \in(-1,1)$, define

$$
\begin{aligned}
H_{f, g}(t) \stackrel{\text { def }}{=} & \mathbb{E}\left[f\left(\frac{1}{\sqrt{2}} G_{1}\right) g\left(\frac{t}{\sqrt{2}} G_{1}+\frac{\sqrt{1-t^{2}}}{\sqrt{2}} G_{2}\right)\right] \\
= & \frac{1}{\pi^{k}\left(1-t^{2}\right)^{k / 2}} \int_{\mathbb{R}^{k}} \int_{\mathbb{R}^{k}} f(x) g(y) \\
& \times \exp \left(\frac{-\|x\|_{2}^{2}-\|y\|_{2}^{2}+2 t\langle x, y\rangle}{1-t^{2}}\right) d x d y .
\end{aligned}
$$

Then $H_{f, g}$ extends to an analytic function on the strip $\{z \in \mathbb{C}: \mathfrak{R}(z) \in(-1,1)\}$. We shall call $\{f, g\}$ a Krivine rounding scheme if $H_{f, g}$ is invertible on a neighborhood of the origin, and if we consider the Taylor expansion

$$
H_{f, g}^{-1}(z)=\sum_{j=0}^{\infty} a_{2 j+1} z^{2 j+1}
$$

then there exists $c=c(f, g) \in(0, \infty)$ satisfying

$$
\sum_{j=0}^{\infty}\left|a_{2 j+1}\right| c^{2 j+1}=1 .
$$


(Only odd Taylor coefficients appear in (2.3) since $H_{f, g}$, and therefore also $H_{f, g}^{-1}$, is odd.)

DEFINITION 2.2 (Alternating Krivine rounding scheme). A Krivine rounding scheme $\{f, g\}$ is called an alternating Krivine rounding scheme if the coefficients $\left\{a_{2 j+1}\right\}_{j=0}^{\infty} \subseteq \mathbb{R}$ in (2.3) satisfy $\operatorname{sign}\left(a_{2 j+1}\right)=(-1)^{j}$ for all $j \in \mathbb{N} \cup\{0\}$. Note that in this case equation (2.4) becomes $H_{f, g}^{-1}(i c) / i=1$, or

$$
c(f, g)=\frac{H_{f, g}(i)}{i} \stackrel{(1.4) \wedge(1.5) \wedge(2.2)}{=} \frac{B_{K}(f, g)}{(\sqrt{2} \pi)^{k}} .
$$

Given a Krivine rounding scheme $f, g: \mathbb{R}^{k} \rightarrow\{-1,1\}$ and $x_{1}, \ldots, x_{m}, y_{1}, \ldots$, $y_{n} \in S^{m+n-1}$, the (generalized) Krivine rounding method proceeds via the following two steps.

Step 1 (Preprocessing the vectors). Consider the Hilbert space

$$
\mathcal{H}=\bigoplus_{j=0}^{\infty}\left(\mathbb{R}^{m+n}\right)^{\otimes(2 j+1)},
$$

where for $k, \ell \in \mathbb{N}$ we set

$$
\left(\mathbb{R}^{k}\right) \stackrel{\otimes \ell}{\stackrel{\text { def }}{=}} \underbrace{\mathbb{R}^{k} \otimes \mathbb{R}^{k} \otimes \cdots \otimes \mathbb{R}^{k}}_{\ell \text { times }} .
$$

For $x \in S^{m+n-1}$, writing $x^{\otimes \ell}$ for the $\ell$-fold tensor product of $x$ with itself, we can then define two vectors $I(x), J(x) \in \mathcal{H}$ by

$$
I(x) \stackrel{\text { def }}{=} \sum_{j=0}^{\infty}\left|a_{2 j+1}\right|^{1 / 2} c^{(2 j+1) / 2} x^{\otimes(2 j+1)}
$$

and

$$
J(x) \stackrel{\text { def }}{=} \sum_{j=0}^{\infty} \operatorname{sign}\left(a_{2 j+1}\right)\left|a_{2 j+1}\right|^{1 / 2} c^{(2 j+1) / 2} x^{\otimes(2 j+1)},
$$

where $c=c(f, g)$. The choice of $c$ was made in order to ensure that $I(x)$ and $J(x)$ are unit vectors in $\mathcal{H}$. Moreover, the definitions (2.6) and (2.7) were made so that the following identity holds:

$$
\forall x, y \in S^{m+n-1}, \quad\langle I(x), J(y)\rangle_{\mathcal{H}}=H_{f, g}^{-1}(c\langle x, y\rangle) .
$$

The preprocessing step of the Krivine rounding method transforms the initial unit vectors $\left\{x_{r}\right\}_{r=1}^{m},\left\{y_{s}\right\}_{s=1}^{n} \subseteq S^{m+n-1}$ to vectors $\left\{u_{r}\right\}_{r=1}^{m},\left\{v_{s}\right\}_{s=1}^{n} \subseteq S^{m+n-1}$ satisfying 
the identities

$$
\begin{aligned}
& \forall(r, s) \in\{1, \ldots, m\} \times\{1, \ldots, n\} \\
& \left\langle u_{r}, v_{s}\right\rangle=\left\langle I\left(x_{r}\right), J\left(y_{s}\right)\right\rangle_{\mathcal{H}} \stackrel{(2.8)}{=} H_{f, g}^{-1}\left(c\left\langle x_{r}, y_{s}\right\rangle\right) .
\end{aligned}
$$

As explained in [2], these new vectors can be computed efficiently provided $H_{f, g}^{-1}$ can be computed efficiently; this simply amounts to computing a Cholesky decomposition.

Step 2 (Random projection). Let $G: \mathbb{R}^{m+n} \rightarrow \mathbb{R}^{k}$ be a random $k \times(m+n)$ matrix whose entries are independent and identically distributed standard Gaussian random variables. Define random signs $\sigma_{1}, \ldots, \sigma_{m}, \tau_{1}, \ldots, \tau_{n} \in\{-1,1\}$ by

$$
\begin{aligned}
& \forall(r, s) \in\{1, \ldots, m\} \times\{1, \ldots, n\} \\
& \quad \sigma_{r} \stackrel{\text { def }}{=} f\left(\frac{1}{\sqrt{2}} G u_{r}\right) \text { and } \tau_{s} \stackrel{\text { def }}{=} g\left(\frac{1}{\sqrt{2}} G v_{s}\right) .
\end{aligned}
$$

Having obtained the random signs $\sigma_{1}, \ldots, \sigma_{m}, \tau_{1}, \ldots, \tau_{n} \in\{-1,1\}$ as in (2.10), for every $m \times n$ matrix $\left(a_{r s}\right)$ we have

$$
\begin{aligned}
\max _{\varepsilon_{1}, \ldots, \varepsilon_{m}, \delta_{1}, \ldots, \delta_{n} \in\{-1,1\}} \sum_{r=1}^{m} \sum_{s=1}^{n} a_{r s} \varepsilon_{r} \delta_{s} & \geqslant \mathbb{E}\left[\sum_{r=1}^{m} \sum_{s=1}^{n} a_{r s} \sigma_{r} \tau_{s}\right] \\
& \stackrel{(\boldsymbol{\alpha})}{=} \sum_{r=1}^{m} \sum_{s=1}^{n} a_{r s} H_{f, g}\left(\left\langle u_{r}, v_{s}\right\rangle\right) \\
& \stackrel{(2.9)}{=} c(f, g) \sum_{r=1}^{m} \sum_{s=1}^{n} a_{r s}\left\langle x_{r}, y_{s}\right\rangle,
\end{aligned}
$$

where (\$) follows by rotation invariance from (2.10) and (2.2). We have thus proved the following corollary, which yields a systematic way to bound the Grothendieck constant from above.

COROllary 2.3. Assume that $f, g: \mathbb{R}^{k} \rightarrow\{-1,1\}$ is a Krivine rounding scheme. Then

$$
K_{G} \leqslant \frac{1}{c(f, g)} .
$$

Krivine's proof of (1.2) corresponds to Corollary 2.3 when $k=1$ and $f(x)=$ $g(x)=\operatorname{sign}(x)$. In this case, $\{f, g\}$ is an alternating Krivine rounding scheme with $H_{f, g}(t)=(2 / \pi) \arcsin (t)$ (Grothendieck's identity). By (2.5), we have $c(f, g)=$ $(2 / \pi i) \arcsin (i)=(2 / \pi) \log (1+\sqrt{2})$, so Corollary 2.3 does indeed correspond to Krivine's bound (1.2). 
One might expect that, since we want to round vectors $x_{1}, \ldots, x_{m}, y_{1}, \ldots, y_{n} \in$ $S^{m+n-1}$ to signs $\varepsilon_{1}, \ldots, \varepsilon_{m}, \delta_{1}, \ldots, \delta_{n} \in\{-1,1\}$, the best possible Krivine rounding scheme occurs when $k=1$ and $f(x)=g(x)=\operatorname{sign}(x)$. This is the intuition leading to König's conjecture. The following simple corollary of Theorem 1.4 says that among all one-dimensional Krivine rounding schemes $f, g: \mathbb{R} \rightarrow\{-1,1\}$ we indeed have $c(f, g) \leqslant c$ (sign, sign), so it does not pay off to take partitions of $\mathbb{R}$ which are more complicated than the half-line partition.

Lemma 2.4. Let $f, g: \mathbb{R} \rightarrow \mathbb{R}$ be a Krivine rounding scheme. Then $c(f, g) \leqslant$ $(2 / \pi) \log (1+\sqrt{2})$.

Proof. Denote $c=c(f, g)$, and assume for contradiction that $c>(2 / \pi) \log (1+$ $\sqrt{2}$ ). Let $r$ be the radius of convergence of the power series of $H_{f, g}^{-1}$ given in (2.3). Due to (2.4), we know that $r \geqslant c>(2 / \pi) \log (1+\sqrt{2})$. Denote

$$
\alpha \stackrel{\text { def }}{=} \frac{H_{f, g}(i)}{i} \stackrel{(1.4) \wedge(1.5) \wedge(2.2)}{=} \frac{B_{K}(f, g)}{\pi \sqrt{2}} .
$$

By Theorem 1.4, we have $|\alpha| \leqslant(2 / \pi) \log (1+\sqrt{2})<r$, and therefore $H_{f, g}^{-1}$ is well defined at the point $i \alpha \in \mathbb{C}$. Thus,

$$
1 \stackrel{(2.11)}{=} \frac{H_{f, g}^{-1}(i \alpha)}{i} \stackrel{(2.3)}{=} \sum_{j=0}^{\infty}(-1)^{j} a_{2 j+1} \alpha^{2 j+1} \leqslant \sum_{j=0}^{\infty}\left|a_{2 j+1}\right| \cdot|\alpha|^{2 j+1} .
$$

By the definition of $c$ in (2.4), we deduce that $c \leqslant|\alpha| \leqslant(2 / \pi) \log (1+\sqrt{2})$, as required.

The conceptual message behind Theorem 1.1 is that, despite the above satisfactory state of affairs in the one-dimensional case, it does pay off to use more complicated higher-dimensional partitions. Specifically, our proof of Theorem 1.1 uses the following rounding procedure. Let $c, p \in(0,1)$ be small enough absolute constants. Given $\left\{x_{r}\right\}_{r=1}^{m},\left\{y_{s}\right\}_{s=1}^{n} \subseteq S^{m+n-1}$, we preprocess them to obtain new vectors $\left\{u_{r}=u_{r}(p, c)\right\}_{r=1}^{m},\left\{v_{s}=v_{s}(p, c)\right\}_{s=1}^{n} \subseteq S^{m+n-1}$. Due to certain technical complications, these new vectors are obtained via a procedure that is similar to the preprocessing step (Step 1) described above, but is not identical to it. We refer to Section 5 for a precise description of the preprocessing step that we use (we conjecture that this complication is unnecessary; see Conjecture 5.7). Once the new vectors $\left\{u_{r}\right\}_{r=1}^{m},\left\{v_{s}\right\}_{s=1}^{n} \subseteq S^{m+n-1}$ have been constructed, we take a $2 \times(m+n)$ matrix $G$ with entries that are independent and identically distributed standard Gaussian random variables, and we consider the random vectors $\left\{G u_{r}=\left(\left(G u_{r}\right)_{1},\left(G u_{r}\right)_{2}\right)\right\}_{r=1}^{m},\left\{G v_{s}=\left(\left(G v_{s}\right)_{1},\left(G v_{s}\right)_{2}\right)\right\}_{s=1}^{n} \subseteq \mathbb{R}^{2}$. Having thus obtained new vectors in $\mathbb{R}^{2}$, with probability $(1-p)$, we 'round' our 
initial vectors to the signs $\left\{\operatorname{sign}\left(\left(G u_{r}\right)_{2}\right)\right\}_{r=1}^{m},\left\{\operatorname{sign}\left(\left(G v_{s}\right)_{2}\right)\right\}_{s=1}^{n} \subseteq \mathbb{R}$, while with probability $p$ we round $x_{r}$ to +1 if

$$
\left(G u_{r}\right)_{2} \geqslant c\left(\left(\left(G u_{r}\right)_{1}\right)^{5}-10\left(\left(G u_{r}\right)_{1}\right)^{3}+15\left(G u_{r}\right)_{1}\right) .
$$

and we round $x_{r}$ to -1 if

$$
\left(G u_{r}\right)_{2}<c\left(\left(\left(G u_{r}\right)_{1}\right)^{5}-10\left(\left(G u_{r}\right)_{1}\right)^{3}+15\left(G u_{r}\right)_{1}\right) .
$$

For concreteness, at this juncture it suffices to describe our rounding procedure without explaining how it was derived: the origin of the fifth-degree polynomial appearing in (2.12) and (2.13) will become clear in Sections 4 and 5. The rounding procedure for $y_{s}$ is identical to (2.12) and (2.13), with $\left(G v_{s}\right)_{1},\left(G v_{s}\right)_{2}$ replacing $\left(G u_{r}\right)_{1},\left(G u_{r}\right)_{2}$, respectively.

\section{The tiger partition and directions for future research}

The partition of the plane described in Figure 1 leads to a proof of Theorem 1.1, but it is not the optimal partition for this purpose. It makes more sense to use the partitions corresponding to maximizers $f_{\max }, g_{\max }: \mathbb{R}^{2} \rightarrow\{-1,1\}$ of König's bilinear form $B_{K}$ as defined in (1.5), that is,

$$
\begin{aligned}
B_{K}\left(f_{\max }, g_{\max }\right)= & \max _{f, g: \mathbb{R}^{2} \rightarrow\{-1,1\}} B_{K}(f, g) \\
= & \max _{f, g: \mathbb{R}^{2} \rightarrow\{-1,1\}} \int_{\mathbb{R}^{2}} \int_{\mathbb{R}^{2}} f(x) g(y) \\
& \times \exp \left(-\frac{\|x\|_{2}^{2}+\|y\|_{2}^{2}}{2}\right) \sin (\langle x, y\rangle) d x d y .
\end{aligned}
$$

A straightforward weak compactness argument shows that the maximum in (3.1) is indeed attained (see Section 6).

Given $f: \mathbb{R}^{2} \rightarrow\{-1,1\}$, define $\sigma(f): \mathbb{R}^{2} \rightarrow\{-1,1\}$ by

$$
\sigma(f)(y) \stackrel{\text { def }}{=} \operatorname{sign}\left(\int_{\mathbb{R}^{2}} f(x) e^{-\|x\|_{2}^{2} / 2} \sin (\langle x, y\rangle) d x\right) .
$$

Then

$$
\sigma\left(f_{\max }\right)=g_{\max } \quad \text { and } \quad \sigma\left(g_{\max }\right)=f_{\max } .
$$

Given $f: \mathbb{R}^{2} \rightarrow\{-1,1\}$, we can then hope to approach $f_{\max }$ by considering the iterates $\left\{\sigma^{2 j}(f)\right\}_{j=1}^{\infty}$. If these iterates converge to $f_{\infty}$, then the pair of functions $\left\{f_{\infty}, \sigma\left(f_{\infty}\right)\right\}$ would satisfy equations (3.2). One can easily check that $\sigma\left(f_{0}\right)=$ $f_{0}$ when $f_{0}: \mathbb{R}^{2} \rightarrow\{-1,1\}$ is given by $f_{0}\left(x_{1}, x_{2}\right)=\operatorname{sign}\left(x_{2}\right)$. But, we have experimentally applied the above iteration procedure to a variety of initial 


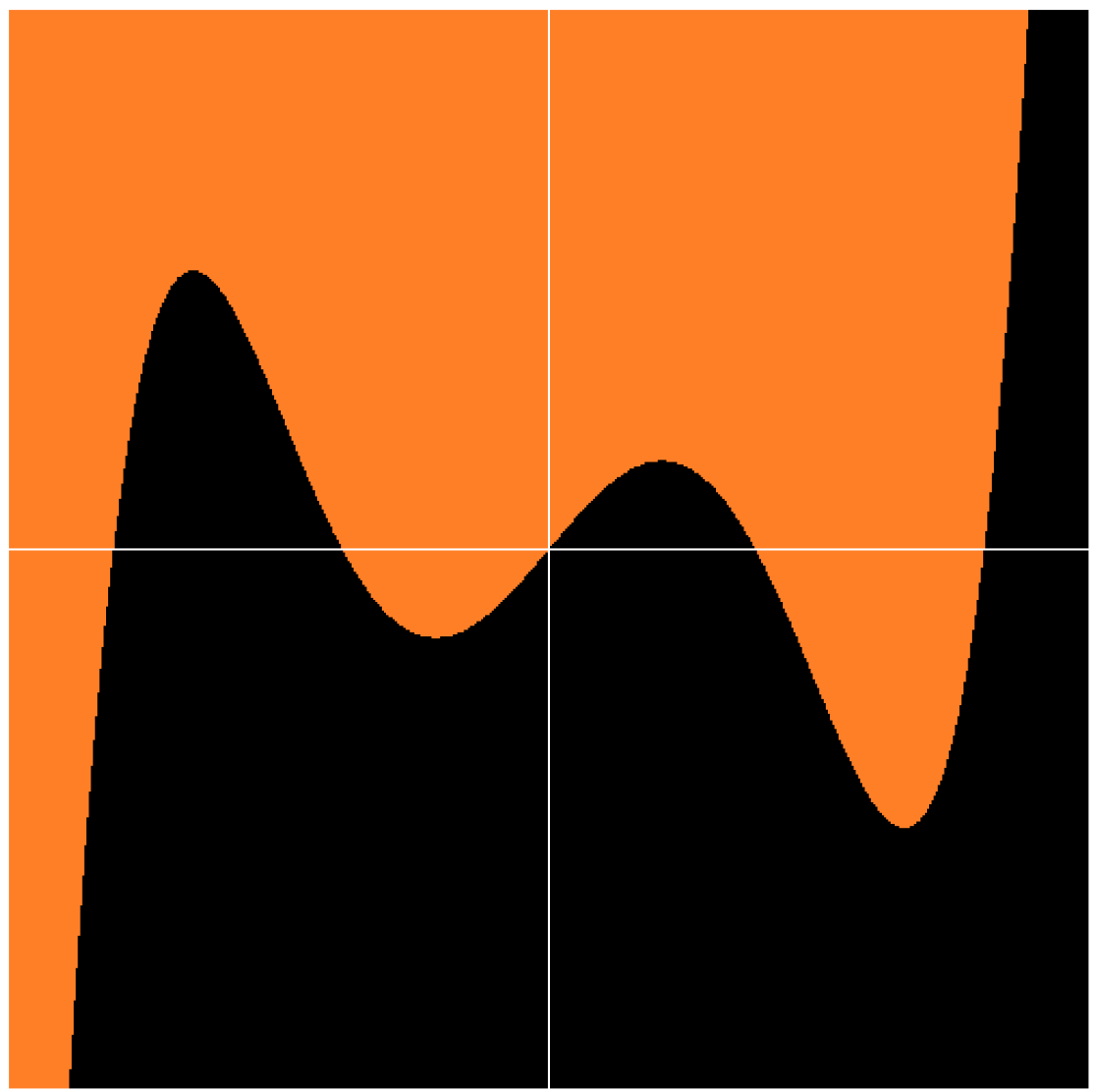

Figure 1. The rounding procedure used in the proof of Theorem 1.1 relies on the partition of $\mathbb{R}^{2}$ depicted above. After a preprocessing step, high-dimensional vectors are projected randomly onto $\mathbb{R}^{2}$ using a matrix with independent and identically distributed standard Gaussian entries. With a certain fixed probability, if the projected vector falls above the graph $y=c\left(x^{5}-10 x^{3}+15 x\right)$ then it is assigned the value +1 , and otherwise it is assigned the value -1 .

functions $f \neq f_{0}$ (both deterministic and random choices), and in all cases the numerical computations suggest that the iterates $\left\{\sigma^{2 j}(f)\right\}_{j=1}^{\infty}$ converge to the function $f_{\infty}$ that is depicted in Figures 2 and 3 (the corresponding function $g_{\infty}=\sigma\left(f_{\infty}\right)$ is different from $f_{\infty}$, but has a similar structure).

QUESTION 3.1. Find an analytic description of the function $f_{\infty}$ from Figures 2 and3. Our numerical computations suggest that the iterates $\left\{\sigma^{2 j}(f)\right\}_{j=1}^{\infty}$ converge to $f_{\infty}$ for (almost?) all initial data $f: \mathbb{R}^{2} \rightarrow\{-1,1\}$ with $f \neq f_{0}$. Can this statement 


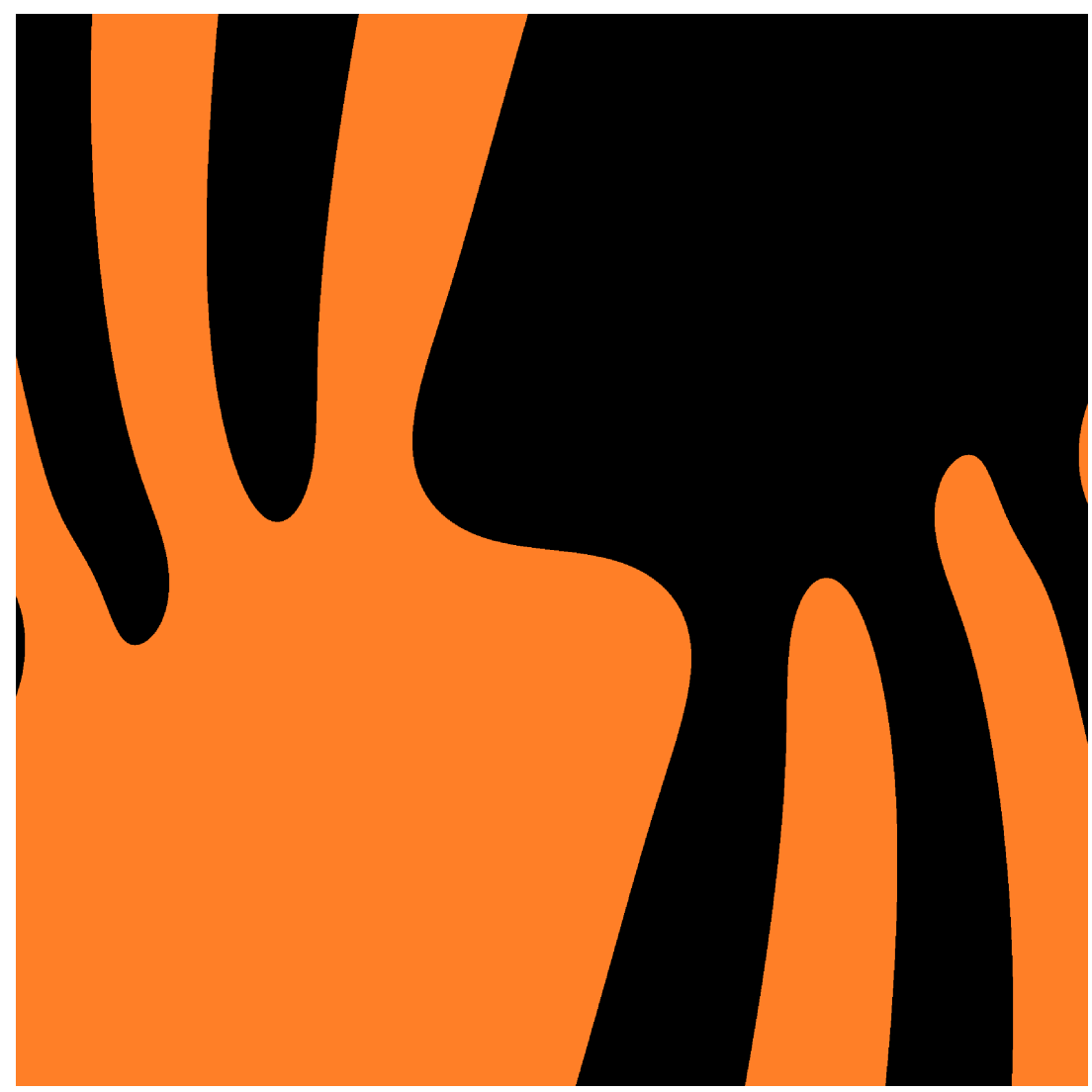

Figure 2. The tiger partition: a depiction of the limiting function $f_{\infty}$ restricted to the square $[-7,7] \times[-7,7] \subseteq \mathbb{R}^{2}$, based on numerical computations. The two shaded regions correspond to the points where $f_{\infty}$ takes the values +1 and -1 .

be made rigorous? If so, is it the case that $\left\{f_{\infty}, \sigma\left(f_{\infty}\right)\right\}$ are maximizers of the bilinear form $B_{K}$ ? We conjecture that the answer to this question is positive.

QUESTION 3.2. Analogously to the above planar computations, can one find an analytic description of the maximizers $f_{\max }^{(n)}, g_{\max }^{(n)}: \mathbb{R}^{n} \rightarrow\{-1,1\}$ of the $n$-dimensional version of König's bilinear form $B_{K}$ ? If so, does $\left\{f_{\max }^{(n)}, g_{\max }^{(n)}\right\}$ form an alternating Krivine rounding scheme (recall Definition 2.2)?

We do not have sufficient data to conjecture whether the answer to Question 3.2 is positive or negative. But we note that, if $\left\{f_{\max }^{(n)}, g_{\max }^{(n)}\right\}$ were an 


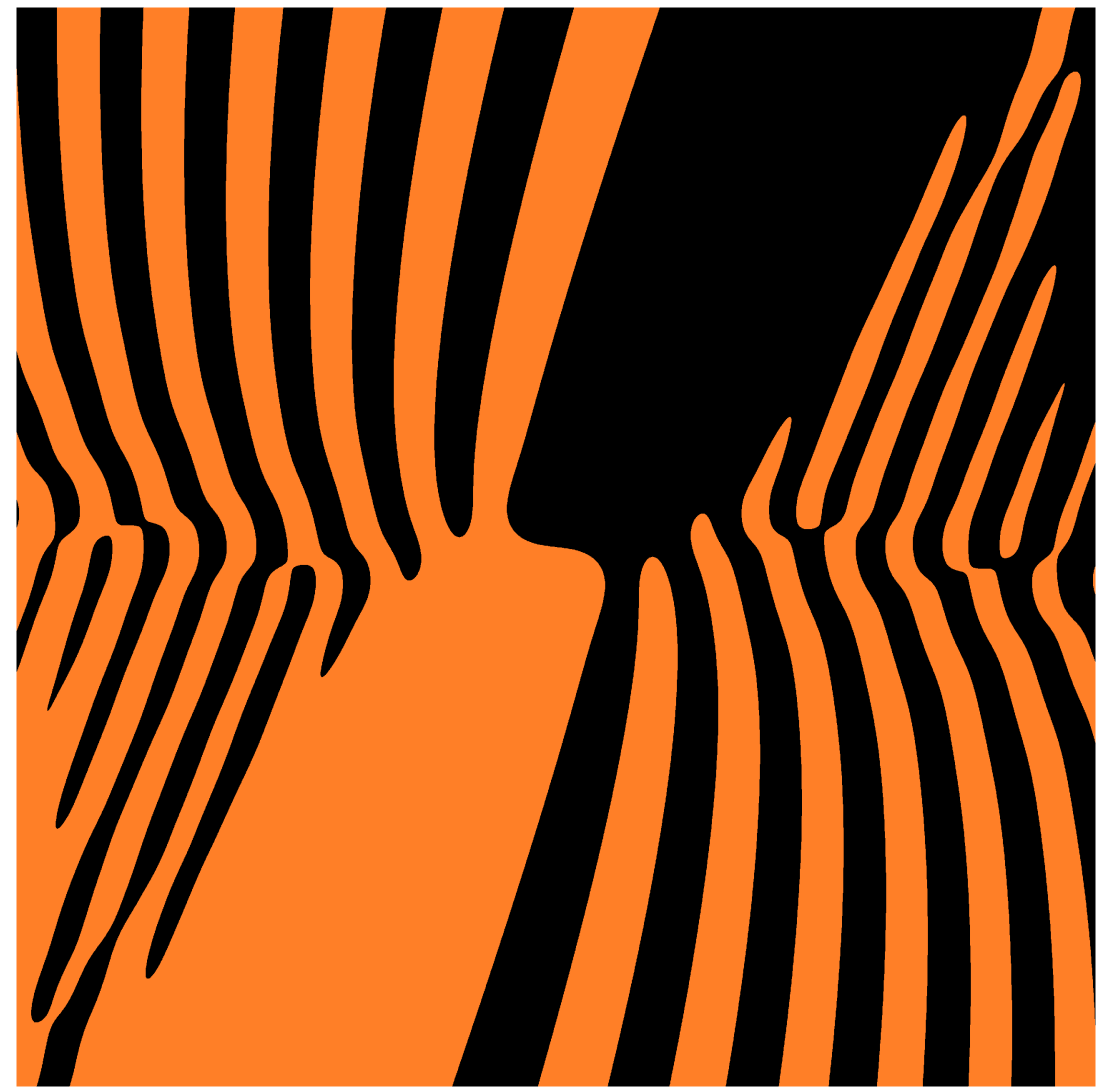

Figure 3. A zoomed-out view of the tiger partition: a depiction of the limiting function $f_{\infty}$ restricted to the square $[-20,20] \times[-20,20] \subseteq \mathbb{R}^{2}$, based on numerical computations.

alternating Krivine rounding scheme, then

$$
K_{G}=\sup _{n \in \mathbb{N}} \frac{(\sqrt{2} \pi)^{n}}{B_{K}\left(f_{\max }^{(n)}, g_{\max }^{(n)}\right)}=\sup _{n \in \mathbb{N}} \frac{(\sqrt{2} \pi)^{n}}{\left\|T_{K}\right\|_{L_{\infty}\left(\mathbb{R}^{n}\right) \rightarrow L_{1}\left(\mathbb{R}^{n}\right)}} .
$$

Indeed, assuming that $\left\{f_{\max }^{(n)}, g_{\max }^{(n)}\right\}$ is an alternating Krivine rounding scheme, the upper bound in (3.3) follows from Corollary 2.3 and the identity (2.5). For the reverse inequality in (3.3), we proceed as in [22]. Using (1.3) with $f, g: \mathbb{R}^{n} \rightarrow S^{n-1}$ given by $f(x)=g(x)=x /\|x\|_{2}$, we see that

$$
K_{G} \geqslant \frac{\int_{\mathbb{R}^{n}} \int_{\mathbb{R}^{n}} K(x, y) \frac{\langle x, y\rangle}{\|x\|_{2}\|y\|_{2}} d x d y}{\left\|T_{K}\right\|_{L_{\infty}\left(\mathbb{R}^{n}\right) \rightarrow L_{1}\left(\mathbb{R}^{n}\right)}},
$$


and we conclude that (3.3) is true since, by equation (2.3) in [22], the integral in the numerator of (3.4) equals $2^{n / 2} \pi^{n}\left(1-1 / n+O\left(1 / n^{2}\right)\right)$.

REMARK 3.3. The series of previously published improvements of the upper bound on the Grothendieck constant highlighted a specific numerical value that was better than the previously known bound. Purposefully departing from this tradition here, we stress that this does not indicate anything unusual or surprising about our work: one can extract an explicit value of $\varepsilon_{0} \in(0,1)$ in Theorem 1.1 by mechanically tracking the constants in the ensuing arguments. We believe that it is important to keep the discussion on a conceptual level and that it would be a mistake to state an explicit new upper bound on $K_{G}$ since it would not have any significance and it would be easily improvable. Specifically, the fifth-degree polynomial appearing in equations (2.12), (2.13) and in Figure 1 was chosen as a simple way to show that two-dimensional rounding methods can improve over Krivine's bound, but by working with polynomials of higher and higher degree one can incrementally improve the bounds that follow from our approach. More significantly, one should work instead with the optimal partition of $\mathbb{R}^{2}$, which we conjecture is the tiger partition. But, this too would not be very meaningful, since we do not see a good reason why two-dimensional partitions are special in the present context. Recently, it was shown in [30] that by working with Krivine-type rounding schemes in $\mathbb{R}^{k}$ one can approach the exact value of the Grothendieck constant as $k \rightarrow \infty$ (here, we use the obvious extension of the previous discussion to probability distributions over partitions of $\mathbb{R}^{k}$ rather than single partitions; note that we work here with random partitions as well, since the polynomial partition of Figure 1 is used with probability $p$ ). It is therefore natural for future work to focus on the asymptotic regime, that is, understanding the structure of the optimal $k$-dimensional partitions for large $k$, while at the present juncture any explicit bound on $\varepsilon_{0}$ would involve an arbitrary choice. In summary, despite the fact that our proof of Theorem 1.1 contains some fairly involved constructions and technical arguments, the true content of our work is conceptual/structural. The Krivine and König conjectures express the belief that certain maximizers have a very simple structure, and here we show that the structure of the maximizers is richer than anticipated. Hopefully, by switching attention to an asymptotic point of view, structural information on the optimal partitions of $\mathbb{R}^{k}$ will emerge as $k \rightarrow \infty$. This challenging research direction might lead to meaningful new numerical bounds on $K_{G}$, while trying to refine numerical estimates based on low-dimensional partitions will most likely distract from the actual problem at hand.

\section{A counterexample to König's conjecture}

In this section, we will make use of several facts on the Hermite polynomials, for which we refer to $\left[3\right.$, Section 6.1]. We let $\left\{h_{m}: \mathbb{R} \rightarrow \mathbb{R}\right\}_{m=0}^{\infty}$ denote the 
sequence of Hermite polynomials normalized so that they form an orthonormal basis with respect to the measure on $\mathbb{R}$ whose density is $x \mapsto e^{-x^{2}}$. Explicitly,

$$
h_{m}(x) \stackrel{\text { def }}{=} \frac{(-1)^{m}}{\sqrt{2^{m} m ! \sqrt{\pi}}} \cdot e^{x^{2}} \frac{d^{m}}{d x^{m}}\left(e^{-x^{2}}\right),
$$

so

$$
\int_{\mathbb{R}} h_{m}(x) h_{k}(x) e^{-x^{2}} d x=\delta_{m k} .
$$

For the correctness of the normalization in (4.1), see equation (6.1.5) in [3].

For reasons that will become clear in Remark 4.5 below, we will consider especially the fifth Hermite polynomial $h_{5}$, in which case (4.1) becomes

$$
h_{5}(x)=\frac{4 x^{5}-20 x^{3}+15 x}{2 \sqrt[4]{\pi} \sqrt{15}} .
$$

We record for future use the following technical lemma.

LEMMA 4.1. The following identities hold true:

$$
\int_{-\infty}^{\infty} e^{-x^{2}} h_{5}(x)^{4} d x=\frac{4653}{\sqrt{\pi}},
$$

and

$$
\int_{-\infty}^{\infty} \int_{-\infty}^{\infty} \exp \left(-\frac{x_{1}^{2}+y_{1}^{2}}{2}\right) h_{5}\left(x_{1}\right)^{2} h_{5}\left(y_{1}\right)^{2} \cos \left(x_{1} y_{1}\right) d x_{1} d y_{1}=49 \sqrt{2}
$$

Proof. We shall use equation (6.1.7) in [3], which asserts that for every $x, t \in \mathbb{R}$ we have

$$
\sum_{n=0}^{\infty} \frac{h_{n}(x)}{\sqrt{n !}} t^{n}=\frac{1}{\sqrt[4]{\pi}} \exp \left(\sqrt{2} t x-\frac{t^{2}}{2}\right) .
$$

It follows that for every $x, u_{1}, u_{2}, u_{3}, u_{4} \in \mathbb{R}$

$$
\begin{gathered}
\left(\sum_{a=0}^{\infty} \frac{h_{a}(x)}{\sqrt{a !}} u_{1}^{a}\right)\left(\sum_{b=0}^{\infty} \frac{h_{b}(x)}{\sqrt{b !}} u_{2}^{b}\right)\left(\sum_{c=0}^{\infty} \frac{h_{c}(x)}{\sqrt{c !}} u_{3}^{c}\right)\left(\sum_{d=0}^{\infty} \frac{h_{d}(x)}{\sqrt{d !}} u_{4}^{d}\right) \\
=\frac{1}{\pi} \exp \left(\sqrt{2} x\left(u_{1}+u_{2}+u_{3}+u_{4}\right)-\frac{u_{1}^{2}+u_{2}^{2}+u_{3}^{2}+u_{4}^{2}}{2}\right) .
\end{gathered}
$$

Note that for every $A \in \mathbb{R}$ we have

$$
\begin{aligned}
\int_{-\infty}^{\infty} \exp \left(-x^{2}+\sqrt{2} A x\right) d x & =e^{A^{2} / 2} \int_{-\infty}^{\infty} \exp \left(-\left(x-\frac{A}{\sqrt{2}}\right)^{2}\right) d x \\
& =\sqrt{\pi} e^{A^{2} / 2}
\end{aligned}
$$


Hence, if we multiply both sides of (4.6) by $e^{-x^{2}}$ and integrate over $x \in \mathbb{R}$, we obtain the following identity:

$$
\begin{array}{r}
\sum_{a=0}^{\infty} \sum_{b=0}^{\infty} \sum_{c=0}^{\infty} \sum_{d=0}^{\infty} \frac{u_{1}^{a} u_{2}^{b} u_{3}^{c} u_{4}^{d}}{\sqrt{a ! b ! c ! d !}} \int_{-\infty}^{\infty} \int_{-\infty}^{\infty} e^{-x^{2}} h_{a}(x) h_{b}(x) h_{c}(x) h_{d}(x) d x \\
\stackrel{(4.6) \wedge(4.7)}{=} \frac{1}{\sqrt{\pi}} \exp \left(\frac{\left(u_{1}+u_{2}+u_{3}+u_{4}\right)^{2}-u_{1}^{2}-u_{2}^{2}-u_{3}^{2}-u_{4}^{2}}{2}\right) .
\end{array}
$$

By equating the coefficients of $u_{1}^{5} u_{2}^{5} u_{3}^{5} u_{4}^{5}$ on both sides of (4.8), we deduce that

$$
\frac{1}{14400} \int_{-\infty}^{\infty} e^{-x^{2} / 2} h_{5}(x)^{4} d x=\frac{517}{1600 \sqrt{\pi}}
$$

implying (4.3).

To prove (4.4), starting from (4.5), we see that for every $x_{1}, y_{1}, u_{1}, u_{2}, v_{1}, v_{2} \in$ $\mathbb{R}$ we have

$$
\begin{aligned}
& \left(\sum_{a=0}^{\infty} \frac{h_{a}\left(x_{1}\right)}{\sqrt{a !}} u_{1}^{a}\right)\left(\sum_{b=0}^{\infty} \frac{h_{b}\left(x_{1}\right)}{\sqrt{b !}} u_{2}^{b}\right)\left(\sum_{c=0}^{\infty} \frac{h_{c}\left(y_{1}\right)}{\sqrt{c !}} v_{1}^{c}\right)\left(\sum_{d=0}^{\infty} \frac{h_{d}\left(y_{1}\right)}{\sqrt{d !}} v_{2}^{d}\right) \\
& =\frac{1}{\pi} \exp \left(\sqrt{2} x_{1}\left(u_{1}+u_{2}\right)+\sqrt{2} y_{1}\left(v_{1}+v_{2}\right)-\frac{u_{1}^{2}+u_{2}^{2}+v_{1}^{2}+v_{2}^{2}}{2}\right) .
\end{aligned}
$$

We next note the following identity: for every $A, B \in \mathbb{R}$ we have

$$
\begin{aligned}
& \int_{-\infty}^{\infty} \int_{-\infty}^{\infty} \exp \left(\sqrt{2} A x+\sqrt{2} B y-\frac{x^{2}+y^{2}}{2}\right) \cos (x y) d x d y \\
& \quad=\sqrt{2} \pi e^{\left(A^{2}+B^{2}\right) / 2} \cos (A B) .
\end{aligned}
$$

We will prove (4.10) in a moment, but let us first assume its validity and see how to complete the proof of (4.4). If we multiply (4.9) by $\exp \left(-\left(x_{1}^{2}+\right.\right.$ $\left.\left.x_{2}^{2}\right) / 2\right) \cos \left(x_{1} y_{1}\right)$, and then integrate with respect to $\left(x_{1}, y_{1}\right) \in \mathbb{R}^{2}$, we obtain the following identity:

$$
\begin{gathered}
\sum_{a=0}^{\infty} \sum_{b=0}^{\infty} \sum_{c=0}^{\infty} \sum_{d=0}^{\infty} \frac{u_{1}^{a} u_{2}^{b} v_{1}^{c} v_{2}^{d}}{\sqrt{a ! b ! c ! d !}} \int_{-\infty}^{\infty} \int_{-\infty}^{\infty} h_{a}\left(x_{1}\right) h_{b}\left(x_{1}\right) h_{c}\left(y_{1}\right) h_{d}\left(y_{1}\right) \cos \left(x_{1} y_{1}\right) d x_{1} d y_{1} \\
\stackrel{(4.9) \wedge(4.10)}{=} \sqrt{2} e^{u_{1} u_{2}+v_{1} v_{2}} \cos \left(\left(u_{1}+u_{2}\right)\left(v_{1}+v_{2}\right)\right) .
\end{gathered}
$$

By equating the coefficients of $u_{1}^{5} u_{2}^{5} v_{1}^{5} v_{2}^{5}$ on both sides of (4.11), we deduce that

$$
\frac{1}{14400} \int_{-\infty}^{\infty} \int_{-\infty}^{\infty} \exp \left(-\frac{x_{1}^{2}+y_{1}^{2}}{2}\right) h_{5}\left(x_{1}\right)^{2} h_{5}\left(y_{1}\right)^{2} \cos \left(x_{1} y_{1}\right) d x_{1} d y_{1}=\frac{49 \sqrt{2}}{14400},
$$

implying (4.4). 
It remains to prove (4.10). Let $I$ denote the integral on the left-hand side of (4.10). The changes of variable $u=x-\sqrt{2} A$ and $v=y-\sqrt{2} B$ yield the following identity:

$$
I=e^{A^{2}+B^{2}} \int_{-\infty}^{\infty} \int_{-\infty}^{\infty} \exp \left(-\frac{u^{2}+v^{2}}{2}\right) \cos ((u+\sqrt{2} A)(v+\sqrt{2} B)) d u d v .
$$

Note that

$$
\begin{aligned}
\cos ((u+\sqrt{2} A)(v+\sqrt{2} B))= & \cos (u(v+\sqrt{2} B)) \cos (\sqrt{2} A(v+\sqrt{2} B)) \\
& -\sin (u(v+\sqrt{2} B)) \sin (\sqrt{2} A(v+\sqrt{2} B)) .(4 .
\end{aligned}
$$

Since $\int_{-\infty}^{\infty} e^{-u^{2} / 2} \sin (u(v+\sqrt{2} B)) d u=0$, it follows that

$$
\begin{aligned}
e^{-A^{2}-B^{2}} I \stackrel{(4.12) \wedge(4.13)}{=} & \int_{-\infty}^{\infty} \int_{-\infty}^{\infty} \exp \left(-\frac{u^{2}+v^{2}}{2}\right) \cos (u(v+\sqrt{2} B)) \\
& \times \cos (\sqrt{2} A(v+\sqrt{2} B)) d u d v
\end{aligned}
$$

Since (see equation (6.1.1) in [3]) for all $x_{1} \in \mathbb{R}$ we have

$$
\int_{-\infty}^{\infty} e^{-y_{1}^{2} / 2} \cos \left(x_{1} y_{1}\right) d y_{1}=\sqrt{2 \pi} e^{-x_{1}^{2} / 2}
$$

equation (4.14) becomes

$$
\begin{aligned}
e^{-A^{2}-B^{2}} I & =\sqrt{2 \pi} \int_{-\infty}^{\infty} \exp \left(-\frac{v^{2}+(v+\sqrt{2} B)^{2}}{2}\right) \cos (\sqrt{2} A(v+\sqrt{2} B)) d v \\
& =\sqrt{2 \pi} \int_{-\infty}^{\infty} \exp \left(-\frac{(\sqrt{2} v+B)^{2}+B^{2}}{2}\right) \cos (\sqrt{2} A(v+\sqrt{2} B)) d v .
\end{aligned}
$$

The change of variable $w=\sqrt{2} v+B$ in (4.16) gives

$$
\begin{aligned}
e^{-A^{2}-B^{2}} I & =\sqrt{\pi} e^{-B^{2} / 2} \int_{-\infty}^{\infty} e^{-w^{2} / 2} \cos (A(w+B)) d w \\
& =\sqrt{\pi} e^{-B^{2} / 2} \int_{-\infty}^{\infty} e^{-w^{2} / 2}(\cos (A w) \cos (A B)-\sin (A w) \sin (A B)) d w \\
& \stackrel{(4.15)}{=} \sqrt{2} \pi e^{-\left(A^{2}+B^{2}\right) / 2} \cos (A B) .
\end{aligned}
$$

This concludes the proof of (4.10), and therefore the proof of Lemma 4.1 is complete. 
For $\eta \in(0,1)$, let $f_{\eta}: \mathbb{R}^{2} \rightarrow\{-1,1\}$ be given by

$$
f_{\eta}\left(x_{1}, x_{2}\right) \stackrel{\text { def }}{=} \begin{cases}1 & x_{2} \geqslant \eta h_{5}\left(x_{1}\right) \\ -1 & x_{2}<\eta h_{5}\left(x_{1}\right)\end{cases}
$$

Note that, since $h_{5}$ is odd, so is $f_{\eta}$ (almost surely). For $z \in \mathbb{C}$ with $|\mathfrak{R}(z)|<1$ we define

$$
H_{\eta}(z) \stackrel{\text { def }}{=} \frac{1}{2 \pi\left(1-z^{2}\right)} \int_{\mathbb{R}^{2} \times \mathbb{R}^{2}} f_{\eta}(x) f_{\eta}(y) \exp \left(\frac{-\|x\|_{2}^{2}-\|y\|_{2}^{2}+2 z\langle x, y\rangle}{1-z^{2}}\right) d x d y .
$$

LEMMA 4.2. $H_{\eta}$ is analytic on the strip

$$
\mathbb{S} \stackrel{\text { def }}{=}\{z \in \mathbb{C}:|\mathfrak{R}(z)|<1\} .
$$

Moreover, for all $a+b i \in \mathbb{S}$ we have

$$
\left|H_{\eta}(a+b i)\right| \leqslant \frac{\pi\left((1+a)^{2}+b^{2}\right)\left((1-a)^{2}+b^{2}\right)}{2\left(1-a^{2}\right) \sqrt{\left(1-a^{2}\right)^{2}+b^{4}+2\left(1+a^{2}\right) b^{2}}} .
$$

Proof. Assume that $a \in(-1,1)$ and $b \in \mathbb{R}$, and write $z=a+b i$. Then

$$
\begin{aligned}
\left|H_{\eta}(z)\right| \leqslant & \frac{1}{2 \pi\left|1-z^{2}\right|} \int_{\mathbb{R}^{2} \times \mathbb{R}^{2}} \exp \left(-\mathfrak{R}\left(\frac{1}{1-z^{2}}\right)\left(\|x\|_{2}^{2}+\|y\|_{2}^{2}\right)\right. \\
& \left.+2 \mathfrak{R}\left(\frac{z}{1-z^{2}}\right)\langle x, y\rangle\right) d x d y \\
= & \frac{1}{2 \pi\left|1-z^{2}\right|} \int_{\mathbb{R}^{2} \times \mathbb{R}^{2}} \exp \left(-\frac{1}{2} \mathfrak{R}\left(\frac{1}{1+z}\right)\|x+y\|_{2}^{2}\right. \\
& \left.-\frac{1}{2} \mathfrak{R}\left(\frac{1}{1-z}\right)\|x-y\|_{2}^{2}\right) d x d y \\
= & \frac{1}{8 \pi\left|1-z^{2}\right|} \int_{\mathbb{R}^{2} \times \mathbb{R}^{2}} \exp \left(-\frac{1}{2} \mathfrak{R}\left(\frac{1}{1+z}\right)\|u\|_{2}^{2}\right. \\
& \left.-\frac{1}{2} \mathfrak{R}\left(\frac{1}{1-z}\right)\|v\|_{2}^{2}\right) d u d v \\
= & \frac{1}{8 \pi\left|1-z^{2}\right|} \cdot \frac{(2 \pi)^{2}}{\mathfrak{R}\left(\frac{1}{1-z}\right) \mathfrak{R}\left(\frac{1}{1+z}\right)} \\
= & \frac{\pi\left((1+a)^{2}+b^{2}\right)\left((1-a)^{2}+b^{2}\right)}{2\left(1-a^{2}\right) \sqrt{\left(1-a^{2}\right)^{2}+b^{4}+2\left(1+a^{2}\right) b^{2}}}
\end{aligned}
$$


where in (4.21) we used the changes of variable $x+y=u$ and $x-y=v$, whose Jacobian equals $\frac{1}{4}$. Since the integral defining $H_{\eta}$ is absolutely convergent on $\mathbb{S}$, the claim follows.

LemmA 4.3. For every $z \in \mathbb{C}$ with $|\Re(z)|<1$ we have $H_{0}(z)=\arcsin (z)$.

Proof. It suffices to prove this for $z \in(0,1)$. Writing $x=\left(x_{1}, x_{2}\right), y=\left(y_{1}, y_{2}\right) \in$ $\mathbb{R}^{2}$, we have

$$
\begin{aligned}
H_{0}(z)= & \left(\frac{1}{2 \pi\left(1-z^{2}\right)} \int_{\mathbb{R} \times \mathbb{R}} \operatorname{sign}\left(x_{2}\right) \operatorname{sign}\left(y_{2}\right) \exp \left(\frac{-x_{2}^{2}-y_{2}^{2}+2 z x_{2} y_{2}}{1-z^{2}}\right) d x_{2} d y_{2}\right) \\
& \cdot\left(\int_{\mathbb{R} \times \mathbb{R}} \exp \left(\frac{-x_{1}^{2}-y_{1}^{2}+2 z x_{1} y_{1}}{1-z^{2}}\right) d x_{1} d y_{1}\right) \\
= & \frac{1-z^{2}}{2 \pi}\left(\int_{\mathbb{R} \times \mathbb{R}} \operatorname{sign}(u) \operatorname{sign}(v) e^{-u^{2}-v^{2}+2 z u v} d u d v\right) \\
& \times\left(\int_{\mathbb{R} \times \mathbb{R}} e^{-(u-z v)^{2}-\left(1-z^{2}\right) v^{2}} d u d v\right),
\end{aligned}
$$

where we used the changes of variable $(u, v)=\left(x_{j}, y_{j}\right) / \sqrt{1-z^{2}}$ for $j \in\{1,2\}$. Now,

$$
\begin{aligned}
\int_{\mathbb{R} \times \mathbb{R}} e^{-(u-z v)^{2}-\left(1-z^{2}\right) v^{2}} d u d v & =\left(\int_{\mathbb{R}} e^{-w^{2}} d w\right)\left(\int_{\mathbb{R}} e^{-\left(1-z^{2}\right) v^{2}} d v\right) \\
& =\frac{\pi}{\sqrt{1-z^{2}}} .
\end{aligned}
$$

Define

$$
\begin{aligned}
\psi(z) & =\int_{\mathbb{R} \times \mathbb{R}} \operatorname{sign}(u) \operatorname{sign}(v) e^{-u^{2}-v^{2}+2 z u v} d u d v \\
& =2 \int_{0}^{\infty} \int_{0}^{\infty} e^{-u^{2}-v^{2}}\left(e^{2 z u v}-e^{-2 z u v}\right) d u d v
\end{aligned}
$$

Passing to the polar coordinates $u=r \cos \theta$ and $v=r \sin \theta$, and then making the change of variable $r=\sqrt{s}$, we see that

$$
\begin{aligned}
\psi(z) & =2 \int_{0}^{\pi / 2} \int_{0}^{\infty} r e^{-r^{2}}\left(e^{z r^{2} \sin (2 \theta)}-e^{-z r^{2} \sin (2 \theta)}\right) d r d \theta \\
& =\int_{0}^{\pi / 2} \int_{0}^{\infty} e^{-s}\left(e^{s z \sin (2 \theta)}-e^{-s z \sin (2 \theta)}\right) d s d \theta \\
& =\int_{0}^{\pi / 2}\left(\frac{1}{1-z \sin (2 \theta)}-\frac{1}{1+z \sin (2 \theta)}\right) d \theta \\
& =\int_{0}^{\pi / 2} \frac{2 z \sin (2 \theta)}{1-z^{2} \sin ^{2}(2 \theta)} d \theta .
\end{aligned}
$$


Due to the identity

$$
\frac{d}{d \theta}\left(\frac{-1}{\sqrt{1-z^{2}}} \arcsin \left(\frac{z \cos (2 \theta)}{\sqrt{1-z^{2} \sin ^{2}(2 \theta)}}\right)\right)=\frac{2 z \sin (2 \theta)}{1-z^{2} \sin ^{2}(2 \theta)},
$$

we conclude that

$$
\psi(z)=\frac{2 \arcsin (z)}{\sqrt{1-z^{2}}} .
$$

Lemma 4.3 now follows from substituting (4.24) and (4.25) into (4.23).

THEOREM 4.4. There exists $\eta_{0}>0$ such that for all $\eta \in\left(0, \eta_{0}\right)$ we have

$$
\frac{H_{\eta}(i)}{i} \in(\log (1+\sqrt{2}), \infty) .
$$

Theorem 4.4 implies that the answer to König's problem is negative. Indeed,

$$
\begin{aligned}
\frac{H_{\eta}(i)}{i} \stackrel{\stackrel{(4.18)}{=}}{ } \frac{1}{4 \pi} \int_{\mathbb{R}^{2} \times \mathbb{R}^{2}} f_{\eta}(x) f_{\eta}(y) \exp \left(-\frac{\|x\|_{2}^{2}+\|y\|_{2}^{2}}{2}\right) \sin (\langle x, y\rangle) d x d y \\
\quad=\frac{B_{K}\left(f_{\eta}, f_{\eta}\right)}{4 \pi} .
\end{aligned}
$$

Since $\arcsin (i)=i \log (1+\sqrt{2})$, it follows from Lemma 4.3 and Theorem 4.4 that for every $\eta \in\left(0, \eta_{0}\right)$ we have $B_{K}\left(f_{\eta}, f_{\eta}\right)>B_{K}\left(f_{0}, f_{0}\right)$. Since $f_{0}\left(x_{1}, x_{2}\right)=\operatorname{sign}\left(x_{2}\right)$, the claimed negative answer to König's problem follows.

Proof of Theorem 4.4. Define $\varphi(\eta)=4 \pi H_{\eta}(i) / i$. The required result will follow once we prove that $\varphi(\eta)=\varphi(0)+1600 \sqrt{2} \eta^{4}+O\left(\eta^{6}\right)$ as $\eta \rightarrow 0$. To this end, since $\varphi$ is even, it suffices to show that $\varphi^{\prime \prime}(0)=0$ and $\varphi^{\prime \prime \prime \prime}(0)=38400 \sqrt{2}$.

Since $h_{5}$ is odd, we have

$$
\begin{aligned}
\varphi(\eta)= & 4 \int_{-\infty}^{\infty} \int_{-\infty}^{\infty} \int_{\eta h_{5}\left(x_{1}\right)}^{\infty} \int_{\eta h_{5}\left(y_{1}\right)}^{\infty} \exp \left(-\frac{x_{1}^{2}+x_{2}^{2}+y_{1}^{2}+y_{2}^{2}}{2}\right) \\
& \times \sin \left(x_{1} y_{1}+x_{2} y_{2}\right) d x_{2} d y_{2} d x_{1} d y_{1} .
\end{aligned}
$$

Define $F: \mathbb{R}^{2} \rightarrow \mathbb{R}$ by

$$
\begin{aligned}
F\left(u_{1}, u_{2}\right) \stackrel{\text { def }}{=} & 4 \int_{-\infty}^{\infty} \int_{-\infty}^{\infty} \int_{u_{1} h_{5}\left(x_{1}\right)}^{\infty} \int_{u_{2} h_{5}\left(y_{1}\right)}^{\infty} \exp \left(-\frac{x_{1}^{2}+x_{2}^{2}+y_{1}^{2}+y_{2}^{2}}{2}\right) \\
& \times \sin \left(x_{1} y_{1}+x_{2} y_{2}\right) d x_{2} d y_{2} d x_{1} d y_{1},
\end{aligned}
$$

so that $\varphi(\eta)=F(\eta, \eta)$. Since $F$ is symmetric, that is, $F\left(u_{1}, u_{2}\right)=F\left(u_{2}, u_{1}\right)$, it follows that

$$
\varphi^{\prime \prime}(0)=2 \frac{\partial^{2} F}{\partial u_{1}^{2}}(0,0)+2 \frac{\partial^{2} F}{\partial u_{1} \partial u_{2}}(0,0)
$$


and

$$
\varphi^{\prime \prime \prime \prime}(0)=2 \frac{\partial^{4} F}{\partial u_{1}^{4}}(0,0)+8 \frac{\partial^{4} F}{\partial u_{1} \partial u_{2}^{3}}(0,0)+6 \frac{\partial^{4} F}{\partial u_{1}^{2} \partial u_{2}^{2}}(0,0) .
$$

Now,

$$
\begin{aligned}
& \frac{\partial F}{\partial u_{1}}\left(u_{1}, u_{2}\right)=-4 \int_{-\infty}^{\infty} \int_{-\infty}^{\infty} \int_{u_{2} h_{5}\left(y_{1}\right)}^{\infty} \exp \left(-\frac{x_{1}^{2}+u_{1}^{2} h_{5}\left(x_{1}\right)^{2}+y_{1}^{2}+y_{2}^{2}}{2}\right) \\
& \text { - } h_{5}\left(x_{1}\right) \sin \left(x_{1} y_{1}+u_{1} h_{5}\left(x_{1}\right) y_{2}\right) d y_{2} d x_{1} d y_{1} \text {. }
\end{aligned}
$$

By differentiation of (4.28) under the integral with respect to $u_{1}$, we see that

$$
\begin{aligned}
\frac{\partial^{2} F}{\partial u_{1}^{2}}(0,0)= & -4 \int_{-\infty}^{\infty} \int_{-\infty}^{\infty} \int_{0}^{\infty} \exp \left(-\frac{x_{1}^{2}+y_{1}^{2}+y_{2}^{2}}{2}\right) h_{5}\left(x_{1}\right)^{2} y_{2} \\
& \times \cos \left(x_{1} y_{1}\right) d y_{2} d x_{1} d y_{1} \\
= & -4 \int_{-\infty}^{\infty} \int_{-\infty}^{\infty} \exp \left(-\frac{x_{1}^{2}+y_{1}^{2}}{2}\right) h_{5}\left(x_{1}\right)^{2} \cos \left(x_{1} y_{1}\right) d x_{1} d y_{1} \\
\stackrel{(4.15)}{=} & -4 \sqrt{2 \pi} \int_{-\infty}^{\infty} e^{-x_{1}^{2}} h_{5}\left(x_{1}\right)^{2} d x_{1} \stackrel{(4.2)}{=}-4 \sqrt{2 \pi} .
\end{aligned}
$$

By differentiation of (4.28) with respect to $u_{2}$, we see that

$$
\begin{aligned}
\frac{\partial^{2} F}{\partial u_{1} \partial u_{2}}\left(u_{1}, u_{2}\right)= & 4 \int_{-\infty}^{\infty} \int_{-\infty}^{\infty} \exp \left(-\frac{x_{1}^{2}+u_{1}^{2} h_{5}\left(x_{1}\right)^{2}+y_{1}^{2}+u_{2}^{2} h_{5}\left(y_{1}\right)^{2}}{2}\right) \\
& \cdot h_{5}\left(x_{1}\right) h_{5}\left(y_{1}\right) \sin \left(x_{1} y_{1}+u_{1} u_{2} h_{5}\left(x_{1}\right) h_{5}\left(x_{2}\right)\right) d x_{1} d y_{1} .
\end{aligned}
$$

Hence,

$$
\frac{\partial^{2} F}{\partial u_{1} \partial u_{2}}(0,0)=4 \int_{-\infty}^{\infty} \int_{-\infty}^{\infty} \exp \left(-\frac{x_{1}^{2}+y_{1}^{2}}{2}\right) h_{5}\left(x_{1}\right) h_{5}\left(y_{1}\right) \sin \left(x_{1} y_{1}\right) d x_{1} d y_{1}
$$

By equation (6.1.15) of [3], we have

$$
\int_{-\infty}^{\infty} e^{-y_{1}^{2} / 2} h_{5}\left(y_{1}\right) \sin \left(x_{1} y_{1}\right) d y_{1}=\sqrt{2 \pi} e^{-x_{1}^{2} / 2} h_{5}\left(x_{1}\right) .
$$

Hence,

$$
\frac{\partial^{2} F}{\partial u_{1} \partial u_{2}}(0,0) \stackrel{(4.31) \wedge(4.32)}{=} 4 \sqrt{2 \pi} \int_{-\infty}^{\infty} e^{-x_{1}^{2}} h_{5}\left(x_{1}\right)^{2} d x_{1} \stackrel{(4.2)}{=} 4 \sqrt{2 \pi} .
$$

By substituting (4.29) and (4.33) into (4.26), we see that $\varphi^{\prime \prime}(0)=0$.

We shall now proceed to compute $\varphi^{\prime \prime \prime \prime}(0)$, using the identity (4.27). By differentiation of (4.28) under the integral with respect to $u_{1}$ three times, we 
see that

$$
\begin{aligned}
\frac{\partial^{4} F}{\partial u_{1}^{4}}(0,0)= & 12 \int_{-\infty}^{\infty} \int_{-\infty}^{\infty} \int_{0}^{\infty} \exp \left(-\frac{x_{1}^{2}+y_{1}^{2}+y_{2}^{2}}{2}\right) \\
& \times \cos \left(x_{1} y_{1}\right) h_{5}\left(x_{1}\right)^{4}\left(y_{2}+\frac{y_{2}^{3}}{3}\right) d y_{2} d x_{1} d y_{1} \\
= & 20 \int_{-\infty}^{\infty} \int_{-\infty}^{\infty} \exp \left(-\frac{x_{1}^{2}+y_{1}^{2}}{2}\right) \cos \left(x_{1} y_{1}\right) h_{5}\left(x_{1}\right)^{4} d x_{1} d y_{1} \\
\stackrel{(4.15)}{=} & 20 \sqrt{2 \pi} \int_{-\infty}^{\infty} e^{-x_{1}^{2}} h_{5}\left(x_{1}\right)^{4} d x_{1} \stackrel{(4.3)}{=} 93060 \sqrt{2} .
\end{aligned}
$$

Now, by differentiation of (4.30) under the integral with respect to $u_{2}$ twice, we obtain the identity

$$
\begin{gathered}
\frac{\partial^{4} F}{\partial u_{1} \partial u_{2}^{3}}(0,0)=-4 \int_{-\infty}^{\infty} \int_{-\infty}^{\infty} \exp \left(-\frac{x_{1}^{2}+y_{1}^{2}}{2}\right) h_{5}\left(y_{1}\right)^{3} h_{5}\left(x_{1}\right) \sin \left(x_{1} y_{1}\right) d x_{1} d y_{1} \\
\stackrel{(4.32)}{=}-4 \sqrt{2 \pi} \int_{-\infty}^{\infty} e^{-y_{1}^{2} h_{5}\left(y_{1}\right)^{4} d y_{1}} \\
\stackrel{(4.3)}{=}-18612 \sqrt{2} .
\end{gathered}
$$

Finally, by differentiation of (4.30) under the integral once with respect to $u_{1}$ and once with respect to $u_{2}$, we see that

$$
\begin{aligned}
\frac{\partial^{4} F}{\partial u_{1}^{2} \partial u_{2}^{2}}(0,0) & =4 \int_{-\infty}^{\infty} \int_{-\infty}^{\infty} \exp \left(-\frac{x_{1}^{2}+y_{1}^{2}}{2}\right) h_{5}\left(x_{1}\right)^{2} h_{5}\left(y_{1}\right)^{2} \cos \left(x_{1} y_{1}\right) d x_{1} d y_{1} \\
& \stackrel{(4.4)}{=} 196 \sqrt{2} .
\end{aligned}
$$

Hence,

$$
\varphi^{\prime \prime \prime \prime}(0) \stackrel{(4.27) \wedge(4.34) \wedge(4.35) \wedge(4.36)}{=} 38400 \sqrt{2} .
$$

REMARK 4.5. Clearly, we did not arrive at the above proof by guessing that the fifth Hermite polynomial $h_{5}$ is the correct choice in (4.17). We arrived at this choice as the simplest member of a general family of ways to perturb the function $\left(x_{1}, x_{2}\right) \mapsto \operatorname{sign}\left(x_{2}\right)$. Since carrying out the analysis of this perturbation procedure in full generality is quite tedious, we chose to present the shorter proof above. Nevertheless, we would like to explain here how we arrived at the choice of $h_{5}$ in (4.17).

Fix two odd functions $\alpha, \beta: \mathbb{R} \rightarrow \mathbb{R}$, and write their Hermite expansion as

$$
\alpha(x)=\sum_{k=0}^{\infty} a_{k} h_{2 k+1}(x) \text { and } \beta(x)=\sum_{k=0}^{\infty} b_{k} h_{2 k+1}(x) .
$$


For $\eta>0$, define $f_{\eta}, g_{\eta}: \mathbb{R}^{2} \rightarrow\{-1,1\}$ by

$$
f_{\eta}\left(x_{1}, x_{2}\right)=\left\{\begin{array}{ll}
1 & x_{2} \geqslant \eta \alpha\left(x_{1}\right), \\
-1 & x_{2}<\eta \alpha\left(x_{1}\right),
\end{array} \quad \text { and } \quad g_{\eta}\left(x_{1}, x_{2}\right)= \begin{cases}1 & x_{2} \geqslant \eta \beta\left(x_{1}\right), \\
-1 & x_{2}<\eta \beta\left(x_{1}\right)\end{cases}\right.
$$

For $z \in \mathbb{C}$ with $|\Re(z)|<1$, define

$$
H_{\eta}(z)=\frac{1}{2 \pi\left(1-z^{2}\right)} \int_{\mathbb{R}^{2} \times \mathbb{R}^{2}} f_{\eta}(x) g_{\eta}(y) \exp \left(\frac{-\|x\|_{2}^{2}-\|y\|_{2}^{2}+2 z\langle x, y\rangle}{1-z^{2}}\right) d x d y .
$$

Thus, our final choice (4.18) corresponds to $\alpha=\beta=h_{5}$.

As in the proof of Theorem 4.4, define $\varphi(\eta)=4 \pi H_{\eta}(i) / i$. In order to show that we have $\varphi(\eta)>\varphi(0)$ for small enough $\eta \in(0,1)$, we start by computing $\varphi^{\prime \prime}(0)$, which turns out to be given by the following formula:

$$
\varphi^{\prime \prime}(0)=-4 \sqrt{2 \pi} \sum_{k=0}^{\infty}\left(a_{k}-(-1)^{k} b_{k}\right)^{2} \leqslant 0 .
$$

Therefore, since $\varphi$ is odd and hence its odd-order derivatives at 0 vanish, in order for us to have a chance to prove that $\varphi(\eta)>\varphi(0)$ for small enough $\eta \in(0,1)$, we must have $\varphi^{\prime \prime}(0)=0$. Due to (4.38), in terms of Hermite coefficients this forces the constraints

$$
\forall k \in \mathbb{N} \cup\{0\}, \quad a_{k}=(-1)^{k} b_{k} .
$$

We can therefore at best hope that $\varphi(\eta)$ is greater than $\varphi(0)$ by a fourth-order term, and we need to compute $\varphi^{\prime \prime \prime \prime}(0)$. This is possible to do, using some identities from [4]. Denote, for $a, b, c, d, k \in \mathbb{N} \cup\{0\}$,

$$
\begin{aligned}
L_{k}(a, b, c, d) \stackrel{\text { def }}{=} & \frac{\sqrt{2(2 a+1) !(2 b+1) !(2 c+1) !(2 d+1) !}}{(2 k) !(a+b+1-k) !(c+d+1-k) !} \\
& \cdot\left(\begin{array}{c}
2 k \\
a-b+k
\end{array}\right)\left(\begin{array}{c}
2 k \\
c-d+k
\end{array}\right),
\end{aligned}
$$

where we use the convention that $L_{k}(a, b, c, d)=0$ whenever there is a negative number in the above factorials or binomial coefficients. A somewhat tedious computation that uses results from [4] shows that if the constraint (4.39) is satisfied then

$$
\varphi^{\prime \prime \prime \prime}(0)=8 \sum_{\substack{(k, p, q, r, s) \in \mathbb{N} \cup\{0\} \\ p+q+r+s \text { is even }}} a_{p} a_{q} a_{r} a_{s} L_{k}(p, q, r, s)\left(1+3(-1)^{k+r+s}\right) .
$$

If, for simplicity, we want to make the choice $\alpha=\beta$, the simplest solution of the constraints (4.39) comes from taking $\alpha=\beta=h_{5}\left(\alpha=\beta=h_{1}\right.$ will not 
work, since then one can check that $\varphi(\eta)=\varphi(0)$ for all $\eta)$. Note, however, that $\alpha=-\beta=h_{3}$ would work here too.

\section{Proof that $K_{G}<\frac{\pi}{2 \log (1+\sqrt{2})}$}

We will fix from now on some $\eta \in\left(0, \eta_{0}\right)$, where $\eta_{0}$ is as in Theorem 4.4. For $p \in[0,1]$, define

$$
F_{p} \stackrel{\text { def }}{=}(1-p) H_{0}+p H_{\eta},
$$

where $H_{\eta}$ is as in (4.18). In what follows, we will denote the unit disk in $\mathbb{C}$ by

$$
\mathbb{D} \stackrel{\text { def }}{=}\{z \in \mathbb{C}:|z|<1\} .
$$

THEOREM 5.1. The exists $p_{0}>0$ such that for all $p \in\left(0, p_{0}\right)$ we have $F_{p}(\mathbb{S}) \supseteq$ $\frac{9}{10} \mathbb{D}$ and $F_{p}^{-1}$ is well defined and analytic on $\frac{9}{10} \mathbb{D}$. Moreover, if we write $F_{p}^{-1}(z)=$ $\sum_{k=1}^{\infty} a_{k}(p) z^{k}$, then there exists $\gamma=\gamma_{p} \in[0, \infty)$ satisfying

$$
\sum_{k=1}^{\infty}\left|a_{k}(p)\right| \gamma^{k}=1
$$

and

$$
\gamma>\log (1+\sqrt{2})=0.88137 \ldots
$$

Note that, since the Hermite polynomial $h_{5}$ is odd, so is $H_{\eta}$. Hence also $F_{p}$ is odd, and therefore $a_{k}(p)=0$ for even $k$.

Assuming Theorem 5.1 for the moment, we will now deduce Theorem 1.1.

Proof of Theorem 1.1. Fix $p \in\left(0, p_{0}\right)$, and let $\gamma>0$ be the constant from Theorem 5.1. Due to (5.1), $\sum_{k=1}^{\infty} a_{k}(p) \gamma^{k}$ converges absolutely, and therefore $F_{p}^{-1}$ is analytic and well defined on $\gamma \mathbb{D}$. For small enough $p$, some of the coefficients $\left\{a_{k}(p)\right\}_{k=1}^{\infty}$ are negative (since the third Taylor coefficient of $H_{0}^{-1}(z)=\sin z$ is negative), implying that for every $r \in[0,1]$ we have

$$
F_{p}^{-1}(r \gamma)=\sum_{k=1}^{\infty} a_{k}(p) r^{k} \gamma^{k} \in(-1,1) \subseteq \mathbb{S} .
$$

Let $\mathcal{H}$ be a Hilbert space. Define two mappings

$$
L_{p}, R_{p}: \mathcal{H} \rightarrow \bigoplus_{k=1}^{\infty} \mathcal{H}^{\otimes k} \stackrel{\text { def }}{=} \mathcal{K}
$$


by

$L_{p}(x) \stackrel{\text { def }}{=} \sum_{k=1}^{\infty} \sqrt{\left|a_{k}(p)\right|} \gamma^{k / 2} x^{\otimes k} \quad$ and $\quad R_{p}(x) \stackrel{\text { def }}{=} \sum_{k=1}^{\infty} \operatorname{sign}\left(a_{k}(p)\right) \sqrt{\left|a_{k}(p)\right|} \gamma^{k / 2} x^{\otimes k}$.

By (5.1), if $\|x\|_{\mathcal{H}}=1$, then $\left\|L_{p}(x)\right\|_{\mathcal{K}}=\left\|R_{p}(x)\right\|_{\mathcal{K}}=1$. Moreover, if $\|x\|_{\mathcal{H}}=$ $\|y\|_{\mathcal{H}}=1$, then

$$
\left\langle L_{p}(x), R_{p}(y)\right\rangle=\sum_{k=1}^{\infty} a_{k}(p) \gamma^{k}\langle x, y\rangle^{k}=F_{p}^{-1}(\gamma\langle x, y\rangle) \stackrel{(5.3)}{\in} \mathbb{S} .
$$

For $N \in \mathbb{N}$, let $G: \mathbb{R}^{N} \rightarrow \mathbb{R}^{2}$ be a $2 \times N$ random matrix with independent and identically distributed standard Gaussian entries. Let $g_{1}, g_{2} \in \mathbb{R}^{2}$ be the first two columns of $G$ (that is, $g_{1}, g_{2}$ are independent and identically distributed standard two-dimensional Gaussian vectors). If $x, y \in \mathbb{R}^{N}$ are unit vectors satisfying $\langle x, y\rangle \in \mathbb{S}$ then by rotation invariance we have

$$
\begin{aligned}
& \mathbb{E}\left[f_{\eta}\left(\frac{1}{\sqrt{2}} G x\right) f_{\eta}\left(\frac{1}{\sqrt{2}} G y\right)\right] \\
& =\mathbb{E}\left[f_{\eta}\left(\frac{g_{1}}{\sqrt{2}}\right) f_{\eta}\left(\langle x, y\rangle \frac{g_{1}}{\sqrt{2}}+\sqrt{1-\langle x, y\rangle^{2}} \frac{g_{2}}{\sqrt{2}}\right)\right] \\
& =\frac{1}{(2 \pi)^{2}} \int_{\mathbb{R}^{2} \times \mathbb{R}^{2}} f_{\eta}\left(\frac{u}{\sqrt{2}}\right) f_{\eta}\left(\langle x, y\rangle \frac{u}{\sqrt{2}}+\sqrt{1-\langle x, y\rangle^{2}} \frac{v}{\sqrt{2}}\right) \\
& \times \exp \left(-\frac{\|u\|_{2}^{2}+\|v\|_{2}^{2}}{2}\right) d u d v \\
& =\frac{2}{\pi} H_{\eta}(\langle x, y\rangle),
\end{aligned}
$$

where we made the changes of variable $u=\sqrt{2} u^{\prime}$ and $v=\left(\sqrt{2} v^{\prime}-\right.$ $\left.\sqrt{2}\langle x, y\rangle u^{\prime}\right) / \sqrt{1-\langle x, y\rangle^{2}}$, whose Jacobian is $4 /\left(1-\langle x, y\rangle^{2}\right)$.

Fix an $m \times n$ matrix $A=\left(a_{i j}\right)$, and let $x_{1}, \ldots, x_{m}, y_{1}, \ldots, y_{n} \in \mathcal{H}$ be unit vectors satisfying

$$
\sum_{i=1}^{m} \sum_{j=1}^{n} a_{i j}\left\langle x_{i}, y_{j}\right\rangle=M \stackrel{\text { def }}{=} \max _{u_{1}, \ldots, u_{m}, v_{1}, \ldots, v_{n} \in S_{\mathcal{H}}} \sum_{i=1}^{m} \sum_{j=1}^{n} a_{i j}\left\langle u_{i}, v_{j}\right\rangle,
$$

where $S_{\mathcal{H}}$ denotes the unit sphere of $\mathcal{H}$. Consider the unit vectors $\left\{L_{p}\left(x_{i}\right)\right\}_{i=1}^{m} \cup$ $\left\{R_{p}\left(y_{j}\right)\right\}_{j=1}^{n}$, which we can think of as residing in $\mathbb{R}^{N}$ for $N=m+n$. By (5.4), we have $\left\langle L_{p}\left(x_{i}\right), R_{p}\left(y_{j}\right)\right\rangle \in \mathbb{S}$ for all $i \in\{1, \ldots, m\}$ and $j \in\{1, \ldots, n\}$, so we may use the identity (5.5) for these vectors. Let $\lambda$ be a random variable satisfying $\operatorname{Pr}[\lambda=1]=p, \operatorname{Pr}[\lambda=0]=1-p$. Assume that $\lambda$ is independent of $G$. Define 
random variables $\varepsilon_{1}, \ldots, \varepsilon_{m}, \delta_{1}, \ldots, \delta_{n} \in\{-1,1\}$ by

$$
\varepsilon_{i}=(1-\lambda) f_{0}\left(\frac{1}{\sqrt{2}} G L_{p}\left(x_{i}\right)\right)+\lambda f_{\eta}\left(\frac{1}{\sqrt{2}} G L_{p}\left(x_{i}\right)\right)
$$

and

$$
\delta_{j}=(1-\lambda) f_{0}\left(\frac{1}{\sqrt{2}} G R_{p}\left(y_{j}\right)\right)+\lambda f_{\eta}\left(\frac{1}{\sqrt{2}} G R_{p}\left(y_{j}\right)\right) .
$$

Then,

$$
\begin{aligned}
& \max _{\sigma_{1}, \ldots, \sigma_{m}, \tau_{1}, \ldots, \tau_{n} \in\{-1,1\}} \sum_{i=1}^{m} \sum_{j=1}^{n} a_{i j} \sigma_{i} \tau_{j} \geqslant \mathbb{E}\left[\sum_{i=1}^{m} \sum_{j=1}^{n} a_{i j} \varepsilon_{i} \delta_{j}\right] \\
& \stackrel{(5.5)}{=} \frac{2}{\pi} \sum_{i=1}^{m} \sum_{j=1}^{n} a_{i j}\left((1-p) H_{0}\left(\left\langle L_{p}\left(x_{i}\right), R_{p}\left(y_{j}\right)\right\rangle\right)+p H_{\eta}\left(\left\langle L_{p}\left(x_{i}\right), R_{p}\left(y_{j}\right)\right\rangle\right)\right) \\
& =\frac{2}{\pi} \sum_{i=1}^{m} \sum_{j=1}^{n} a_{i j} F_{p}\left(\left\langle L_{p}\left(x_{i}\right), R_{p}\left(y_{j}\right)\right\rangle\right) \\
& \stackrel{(5.4)}{=} \frac{2}{\pi} \sum_{i=1}^{m} \sum_{j=1}^{n} a_{i j} F_{p}\left(F_{p}^{-1} \gamma\left\langle x_{i}, y_{j}\right\rangle\right) \stackrel{(5.6)}{=} \frac{2 \gamma}{\pi} M .
\end{aligned}
$$

This gives the bound $K_{G} \leqslant \pi / 2 \gamma \stackrel{(5.2)}{<} \pi / 2 \log (1+\sqrt{2})$, as required.

Our goal from now on will be to prove Theorem 5.1.

LEMMA 5.2. $H_{0}$ is one-to-one on $\mathbb{S}$ and $H_{0}(\mathbb{S}) \supseteq \mathbb{D}$.

Proof. The fact that $H_{0}$ is one-to-one on $\mathbb{S}$ is a consequence of Lemma 4.3. To show that $H_{0}(\mathbb{S}) \supseteq \mathbb{D}$, we need to prove that if $a, b \in \mathbb{R}$ and $a^{2}+b^{2}<1$ then $|\Re(\sin (a+b i))|<1$. Now,

$$
|\mathfrak{R}(\sin (a+b i))|=\frac{e^{b}+e^{-b}}{2}|\sin a| .
$$

Using the inequality $|\sin a| \leqslant|a|$, we see that it suffices to show that for all $x \in(0,1)$ we have

$$
\frac{e^{x}+e^{-x}}{2} \sqrt{1-x^{2}}<1
$$

By Taylor's formula, we know that there exists $y \in[0, x]$ such that

$$
\begin{aligned}
\frac{e^{x}+e^{-x}}{2} & =1+\frac{x^{2}}{2}+\frac{x^{4}}{24} \cdot \frac{e^{y}+e^{-y}}{2} \leqslant 1+\frac{x^{2}}{2}+\frac{x^{4}}{24} \cdot \frac{e+e^{-1}}{2} \\
& <1+\frac{x^{2}}{2}+\frac{x^{4}}{12} .
\end{aligned}
$$


Note that

$$
\left(1+\frac{x^{2}}{2}+\frac{x^{4}}{12}\right)^{2}\left(1-x^{2}\right)=1-\frac{7 x^{4}}{12}-\frac{x^{6}}{3}-\frac{11 x^{8}}{144}-\frac{x^{10}}{144}<1,
$$

which together with (5.9) implies (5.8).

REMARK 5.3. A more careful analysis of the expression (5.7) shows that there exists $\varepsilon_{0}>0$ (for example, $\varepsilon_{0}=0.05$ works) such that $H_{0}(\mathbb{S}) \supseteq\left(1+\varepsilon_{0}\right) \mathbb{D}$. Since we will not need this stronger fact here, we included the above simpler proof of a weaker statement.

LEMMA 5.4. For every $r \in(0,1)$ there exists $p_{r} \in(0,1)$ and a bounded open subset $\Omega_{r} \subseteq \mathbb{S}$ with $\overline{\Omega_{r}} \subseteq \mathbb{S}$ such that for all $p \in\left(0, p_{r}\right)$ the function $F_{p}$ is one-to-one on $\Omega_{r}$ and $F_{p}\left(\Omega_{r}\right)=r \mathbb{D}$. Thus $F_{p}^{-1}$ is well defined and analytic on $r \mathbb{D}$.

Proof. For $n \in \mathbb{N}$, consider the set

$$
E_{n}=\left\{z \in \mathbb{C}:|\Re(z)|<1-\frac{1}{n} \wedge|\Im(z)|<n\right\} .
$$

Using Lemma 5.2, fix a large enough $n \in \mathbb{N}$ so that $H_{0}\left(E_{n}\right) \supseteq r \overline{\mathbb{D}}$. The bound (4.20) implies that there exists $M>0$ such that $\left|H_{\eta}(z)\right| \leqslant M$ for all $\eta>0$ and $z \in \partial E_{n+1}$. By Lemma 5.2, $H_{0}$ takes a value $\zeta \in r \overline{\mathbb{D}}$ exactly once on $E_{n+1}$, and this occurs at some point in $E_{n}$. Hence,

$$
m \stackrel{\text { def }}{=} \min _{\substack{\zeta \in \mathbb{D} \\ z \in \partial E_{n+1}}}\left|H_{0}(z)-\zeta\right|>0 .
$$

Define $p_{r}=m /(2 M)$.

Fix $\zeta \in r \mathbb{D}$. If $p \in\left(0, p_{r}\right)$, then for every $z \in \partial E_{n+1}$ we have

$$
\left|p\left(H_{\eta}(z)-H_{0}(z)\right)\right|<\frac{m}{2 M}\left(\left|H_{\eta}(z)\right|+\left|H_{0}(z)\right|\right) \leqslant m \leqslant\left|H_{0}(z)-\zeta\right| .
$$

Rouché's theorem now implies that the number of zeros of $H_{0}-\zeta$ in $E_{n+1}$ is the same as the number of zeros of $H_{0}-\zeta+p\left(H_{\eta}-H_{0}\right)=F_{p}-\zeta$ in $E_{n+1}$. Hence $F_{p}$ takes the value $\zeta$ exactly once in $E_{n+1}$. Since $\zeta$ was an arbitrary point in $r \mathbb{D}$, we can define $\Omega_{r}=F_{p}^{-1}(r \mathbb{D})$.

Lemma 5.5. For every $r \in(0,1)$, there exists $C_{r} \in(0, \infty)$ such that, using the notation of Lemma 5.4, for every $p \in\left(0, p_{r}\right)$ and $z \in r \mathbb{D}$ we have

$$
\left|F_{p}^{-1}(z)-\sin z-p\left(z-H_{\eta}(\sin z)\right) \cos z\right| \leqslant C_{r} p^{2} .
$$


Proof. Note that

$$
z=F_{p}\left(F_{p}^{-1}(z)\right)=(1-p) H_{0}\left(F_{p}^{-1}(z)\right)+p H_{\eta}\left(F_{p}^{-1}(z)\right) .
$$

By differentiating (5.11) with respect to $p$, we see that

$$
\begin{aligned}
0= & H_{\eta}\left(F_{p}^{-1}(z)\right)-H_{0}\left(F_{p}^{-1}(z)\right) \\
& +\left(\frac{d}{d p} F_{p}^{-1}(z)\right)\left((1-p) \frac{d H_{0}}{d z}\left(F_{p}^{-1}(z)\right)+p \frac{d H_{\eta}}{d z}\left(F_{p}^{-1}(z)\right)\right) \\
= & H_{\eta}\left(F_{p}^{-1}(z)\right)-H_{0}\left(F_{p}^{-1}(z)\right)+\left(\frac{d}{d p} F_{p}^{-1}(z)\right) \frac{d F_{p}}{d z}\left(F_{p}^{-1}(z)\right) \\
= & H_{\eta}\left(F_{p}^{-1}(z)\right)-H_{0}\left(F_{p}^{-1}(z)\right)+\frac{\frac{d}{d p} F_{p}^{-1}(z)}{\frac{d}{d z}\left(F_{p}^{-1}(z)\right)} .
\end{aligned}
$$

Hence,

$$
\frac{d}{d p} F_{p}^{-1}(z)=\left(H_{0}\left(F_{p}^{-1}(z)\right)-H_{\eta}\left(F_{p}^{-1}(z)\right)\right) \frac{d}{d z}\left(F_{p}^{-1}(z)\right) .
$$

If we now differentiate (5.12) with respect to $p$, while using (5.12) whenever the term $(d / d p) F_{p}^{-1}(z)$ appears, we obtain the following identity.

$$
\begin{aligned}
\frac{d^{2}}{d p^{2}} F_{p}^{-1}(z)= & {\left[\frac{d H_{0}}{d z}\left(F_{p}^{-1}(z)\right)-\frac{d H_{\eta}}{d z}\left(F_{p}^{-1}(z)\right)+\left(H_{0}\left(F_{p}^{-1}(z)\right)\right.\right.} \\
& \left.\left.-H_{\eta}\left(F_{p}^{-1}(z)\right)\right) \frac{d^{2}}{d z^{2}}\left(F_{p}^{-1}(z)\right)\right] \\
& \cdot\left(H_{0}\left(F_{p}^{-1}(z)\right)-H_{\eta}\left(F_{p}^{-1}(z)\right)\right) \frac{d}{d z}\left(F_{p}^{-1}(z)\right) .
\end{aligned}
$$

Take $M=M_{r}>0$ such that for all $w \in \Omega_{r}$ we have

$$
\max \left\{\left|H_{0}(w)\right|,\left|H_{\eta}(w)\right|,\left|\frac{d H_{0}}{d z}(w)\right|,\left|\frac{d H_{\eta}}{d z}(w)\right|\right\} \leqslant M .
$$

Note that (5.14) applies to $w=F_{p}^{-1}(z)$ for $z \in r \mathbb{D}$. We also define

$$
R=R_{r}=\max _{w \in \partial \Omega_{(1+r) / 2}}|w| .
$$

Then, for $\zeta \in((1+r) / 2) \mathbb{D}$, we have $\left|F_{p}^{-1}(\zeta)\right| \leqslant R$. If $z \in r \mathbb{D}$, then by the Cauchy formula we have

$$
\begin{aligned}
\left|\frac{d}{d z}\left(F_{p}^{-1}(z)\right)\right| & =\left|\frac{1}{\pi(r+1) i} \oint_{((1+r) / 2) \partial \mathbb{D}} \frac{F_{p}^{-1}(\zeta)}{(\zeta-z)^{2}} d \zeta\right| \leqslant \max _{\zeta \in((1+r) / 2) \partial \mathbb{D}} \frac{\left|F_{p}^{-1}(\zeta)\right|}{(|\zeta|-|z|)^{2}} \\
& \leqslant \frac{4 R}{(1-r)^{2}}
\end{aligned}
$$


Similarly,

$$
\left|\frac{d^{2}}{d z^{2}} F_{p}^{-1}(z)\right|=\left|\frac{2}{\pi(r+1) i} \oint_{((1+r) / 2) \partial \mathbb{D}} \frac{F_{p}^{-1}(\zeta)}{(\zeta-z)^{3}} d \zeta\right| \leqslant \frac{16 R}{(1-r)^{3}} .
$$

These estimates, in conjunction with the identity (5.13), imply the following bound:

$$
\left|\frac{d^{2}}{d p^{2}} F_{p}^{-1}(z)\right| \leqslant\left(2 M+2 M \frac{16 R}{(1-r)^{3}}\right) 2 M \frac{4 R}{(1-r)^{2}} .
$$

By the Taylor formula, we deduce that

$$
\left|F_{p}^{-1}(z)-F_{0}^{-1}(z)-p \frac{d}{d p} F_{p}^{-1}(z)\right|_{p=0} \mid \leqslant C_{r} p^{2},
$$

where $C_{r}=\left(8 M^{2} R /(1-r)^{2}\right)\left(1+16 R /(1-r)^{3}\right)$. It remains to note that, due to Lemma 4.3 and the identity (5.12), we have $\left.(d / d p) F_{p}^{-1}(z)\right|_{p=0}=(z-$ $\left.H_{\eta}(\sin z)\right) \cos z$.

Proof of Theorem 5.1. We will fix from now on some $r \in(9 / 10,1)$. For $z \in r \mathbb{D}$, write $\phi(z)=\left(z-H_{\eta}(\sin z)\right) \cos z$. Consider the power series expansions

$$
\sin z=\sum_{k=0}^{\infty} b_{2 k+1} z^{2 k+1}=\sum_{k=0}^{\infty} \frac{(-1)^{k}}{(2 k+1) !} z^{2 k+1},
$$

and

$$
\phi(z)=\sum_{k=0}^{\infty} c_{2 k+1} z^{2 k+1}
$$

By the Cauchy formula, we have, for every $k \in \mathbb{N} \cup\{0\}$,

$$
\left|a_{2 k+1}(p)-b_{2 k+1}-p c_{2 k+1}\right|=\left|\frac{1}{2 \pi i r} \oint_{r \geqslant \mathbb{D}} \frac{F_{p}^{-1}(z)-\sin z-p \phi(z)}{z^{2 k+2}} d z\right| \stackrel{(5.10)}{\leqslant} \frac{C_{r} p^{2}}{r^{2 k+2}} .
$$

Note that by Lemma 4.2 the radius of convergence of the series in (5.15) is at least 1 , and therefore $\sum_{k=0}^{\infty}\left|c_{2 k+1}\right|(9 / 10)^{2 k+1}<\infty$. Hence,

$$
\begin{aligned}
\sum_{k=0}^{\infty}\left|a_{2 k+1}(p)\right|\left(\frac{9}{10}\right)^{2 k+1} \stackrel{(5.16)}{\geqslant} \sum_{k=0}^{\infty} \frac{(9 / 10)^{2 k+1}}{(2 k+1) !}-p \sum_{k=0}^{\infty}\left|c_{2 k+1}\right|\left(\frac{9}{10}\right)^{2 k+1} \\
-\frac{C_{r} p^{2}}{r} \sum_{k=0}^{\infty}\left(\frac{9}{10 r}\right)^{2 k+1} \\
=\frac{e^{9 / 10}-e^{-9 / 10}}{2}-O(p)>1.02-O(p) .
\end{aligned}
$$


By continuity, it follows from (5.17) that, provided $p$ is small enough, there exists $\gamma>0$ satisfying the identity (5.1). Our goal is to prove (5.2), so assume for contradiction that $\gamma \leqslant \log (1+\sqrt{2})<9 / 10$. Note that since $r \in(9 / 10,1)$ we have

$$
\frac{\gamma}{r} \leqslant \frac{10 \log (1+\sqrt{2})}{9}<\frac{49}{50}
$$

Fix $\varepsilon>0$ that will be determined later. We have seen in Lemma 5.2 that $\sin \left(\frac{9}{10} \mathbb{D}\right) \subseteq \mathbb{S}$. Since $H_{\eta}$ is analytic on $\mathbb{S}$, it follows that $\phi$ is analytic on $\frac{9}{10} \mathbb{D}$. Since $\gamma<9 / 10$, there exists $n \in \mathbb{N}$ satisfying

$$
\sum_{k=n+1}^{\infty}\left|c_{2 k+1}\right| \gamma^{2 k+1}<\frac{\varepsilon}{2}
$$

There exists $p=p(\varepsilon)$ such that for all $p \in(0, p(\varepsilon))$ we have $p\left|c_{2 k+1}\right|<\frac{1}{2}\left|b_{2 k+1}\right|$ for all $k \in\{0, \ldots, n\}$. In particular, we have $\operatorname{sign}\left(b_{2 k+1}+p c_{2 k+1}\right)=\operatorname{sign}\left(b_{2 k+1}\right)=$ $(-1)^{k}$. Now,

$$
\begin{aligned}
\left|1-\frac{F_{p}^{-1}(i \gamma)}{i}\right| \stackrel{(5.1)}{=}\left|\sum_{k=0}^{\infty}\left(\left|a_{2 k+1}(p)\right|-(-1)^{k} a_{2 k+1}(p)\right) \gamma^{2 k+1}\right| & \stackrel{(5.16)}{\leqslant} \sum_{k=0}^{\infty}|| b_{2 k+1}+p c_{2 k+1}\left|-(-1)^{k}\left(b_{2 k+1}+p c_{2 k+1}\right)\right| \gamma^{2 k+1} \\
& +2 \sum_{k=0}^{\infty} \frac{C_{r} p^{2}}{r^{2 k+2}} \gamma^{2 k+1}
\end{aligned}
$$

To estimate the two terms on the right-hand side of (5.20), note first that

$$
2 \sum_{k=0}^{\infty} \frac{C_{r} p^{2}}{r^{2 k+2}} \gamma^{2 k+1} \stackrel{(5.18)}{\leqslant} \frac{2 C_{r}}{r} p^{2} \sum_{k=0}^{\infty}\left(\frac{49}{50}\right)^{2 k+1} \leqslant C_{r}^{\prime} p^{2},
$$

where $C_{r}^{\prime}$ depends only on $r$. Since $p \in(0, p(\varepsilon))$, we know that for all $k \in$ $\{0, \ldots, n\}$ we have $\left|b_{2 k+1}+p c_{2 k+1}\right|=(-1)^{k}\left(b_{2 k+1}+p c_{2 k+1}\right)$. Hence the first $n$ terms of the first sum on the right-hand side of (5.20) vanish. Therefore,

$$
\begin{aligned}
& \sum_{k=0}^{\infty}|| b_{2 k+1}+p c_{2 k+1}\left|-(-1)^{k}\left(b_{2 k+1}+p c_{2 k+1}\right)\right| \gamma^{2 k+1} \\
& \quad=\sum_{k=n+1}^{\infty}|| b_{2 k+1}+p c_{2 k+1}|-| b_{2 k+1}\left|-(-1)^{k} p c_{2 k+1}\right| \gamma^{2 k+1} \\
& \quad \leqslant 2 p \sum_{k=n+1}^{\infty}\left|c_{2 k+1}\right| \gamma^{2 k+1} \stackrel{(5.19)}{<} p \varepsilon .
\end{aligned}
$$


By substituting (5.21) and (5.22) into (5.20), we see that, if we define $\beta=$ $F_{p}^{-1}(i \gamma)-i$, then

$$
|\beta| \leqslant C_{r}^{\prime} p^{2}+p \varepsilon
$$

Let $L_{0}$ be the Lipschitz constant of $H_{0}$ on $i+\frac{1}{2} \mathbb{D} \subseteq \mathbb{S}$ (the disc of radius $\frac{1}{2}$ centered at $i$ ). Similarly, let $L_{\eta}$ be the Lipschitz constant of $H_{\eta}$ on $i+\frac{1}{2} \mathbb{D}$, and set $L=\max \left\{L_{0}, L_{\eta}\right\}$. It follows that $F_{p}=(1-p) H_{0}+p H_{\eta}$ is $L$-Lipschitz on $i+\frac{1}{2} \mathbb{D}$. Due to (5.23), if $p$ is small enough then $i+\beta \in i+\frac{1}{2} \mathbb{D}$, and, therefore,

$$
\begin{aligned}
\log (1+\sqrt{2}) \geqslant \gamma & =\frac{F_{p}(\beta+i)}{i} \geqslant \frac{F_{p}(i)}{i}-L|\beta| \\
& \stackrel{(5.23)}{\geqslant} \frac{(1-p) H_{0}(i)+p H_{\eta}(i)}{i}-L p\left(C_{r}^{\prime} p+\varepsilon\right) \\
& =(1-p) \log (1+\sqrt{2})+p \frac{H_{\eta}(i)}{i}-\operatorname{Lp}\left(C_{r}^{\prime} p+\varepsilon\right) .
\end{aligned}
$$

This simplifies to give the following estimate:

$$
\frac{H_{\eta}(i)}{i} \leqslant \log (1+\sqrt{2})+L C_{r}^{\prime} p+L \varepsilon .
$$

Since this is supposed to hold for all $\varepsilon>0$ and $p \in(0, p(\varepsilon))$, we arrive at a contradiction to Theorem 4.4.

REMARK 5.6. An inspection of the proof of Theorem 1.1 shows that the only property of $H_{\eta}$ that was used is that it is a counterexample to König's problem. In other words, assume that $f, g: \mathbb{R}^{2} \rightarrow\{-1,1\}$ are measurable functions, and consider the function $H: \mathbb{S} \rightarrow \mathbb{C}$ given by

$$
H(z)=\frac{1}{2 \pi\left(1-z^{2}\right)} \int_{\mathbb{R}^{2} \times \mathbb{R}^{2}} f(x) g(y) \exp \left(\frac{-\|x\|_{2}^{2}-\|y\|_{2}^{2}+2 z\langle x, y\rangle}{1-z^{2}}\right) d x d y .
$$

Assume that $B_{K}(f, g)>4 \pi \log (1+\sqrt{2})$, where $B_{K}$ is König's bilinear form given in (1.5). Then one can repeat the proof of Theorem 1.1 with $H_{\eta}$ replaced by $H$, arriving at the same conclusion.

Conjecture 5.7. Recalling Definition 2.1 and Corollary 2.3, we conjecture that, for small enough $\eta \in(0,1)$, the pair of functions $f=g=f_{\eta}: \mathbb{R}^{2} \rightarrow\{-1,1\}$ is a Krivine rounding scheme for which we have $c\left(f_{\eta}, f_{\eta}\right)>(2 / \pi) \log (1+\sqrt{2})$. In other words, we conjecture that in order to prove Theorem 1.1 we do not need to use a convex combination of $H_{0}$ and $H_{\eta}$ as we did above, but rather use only $H_{\eta}$ itself. 


\section{Proof of Theorem 1.4}

For the final equality in (1.6), see equation (2.4) in [22]. Denote

$$
\begin{aligned}
M & \stackrel{\text { def }}{=} \sup _{\substack{f, g \in L_{\infty}(0, \infty) \\
f, g \in[-1,1] \text { almosteverywhere }}} \int_{0}^{\infty} \int_{0}^{\infty} f(x) g(y) \exp \left(-\frac{x^{2}+y^{2}}{2}\right) \sin (x y) d x d y \\
& =\sup _{\substack{f, g \in \infty \infty(0, \infty) \\
f, g \in\{-1,1\} \text { almosteverywhere }}} \int_{0}^{\infty} \int_{0}^{\infty} f(x) g(y) \exp \left(-\frac{x^{2}+y^{2}}{2}\right) \sin (x y) d x d(6.1)
\end{aligned}
$$

Theorem 1.4 will follow once we show that $M$ is attained when $f=g=1$ or $f=g=-1$. Note that the supremum in (6.1) is attained at some $f, g:(0, \infty) \rightarrow\{-1,1\}$. Indeed, let $\mu$ be the measure on $(0, \infty)$ with density $x \mapsto$ $e^{-x^{2} / 2}$. If $f_{n}, g_{n}:(0, \infty) \rightarrow[-1,1]$ satisfy $\lim _{n \rightarrow \infty} \int_{0}^{\infty} \int_{0}^{\infty} f_{n}(x) g_{n}(y) \exp \left(-\left(x^{2}+\right.\right.$ $\left.\left.y^{2}\right) / 2\right) \sin (x y) d x d y=M$, then by passing to a subsequence we may assume that there are $f, g \in L_{2}(\mu)$ such that $f_{n}$ converges weakly to $f$ and $g_{n}$ converges weakly to $g$ in $L_{2}(\mu)$. Then $f, g \in[-1,1]$ almost everywhere, and by weak convergence we have $\int_{0}^{\infty} \int_{0}^{\infty} f(x) g(y) \exp \left(-\left(x^{2}+y^{2}\right) / 2\right) \sin (x y) d x d y=M$. A simple extreme point argument shows that we may also assume that $f, g \in\{-1,1\}$.

Assume from now on that $f, g:(0, \infty) \rightarrow\{-1,1\}$ are maximizers of $(6.1)$, that is,

$$
M=\int_{0}^{\infty} \int_{0}^{\infty} f(x) g(y) \exp \left(-\frac{x^{2}+y^{2}}{2}\right) \sin (x y) d x d y .
$$

This implies that for almost every $x \in(0, \infty)$ we have

$$
f(x)=\operatorname{sign}\left(\int_{0}^{\infty} g(y) e^{-y^{2} / 2} \sin (x y) d y\right),
$$

and

$$
g(x)=\operatorname{sign}\left(\int_{0}^{\infty} f(y) e^{-y^{2} / 2} \sin (x y) d y\right) .
$$

Consequently,

$$
\begin{aligned}
M & =\int_{0}^{\infty}\left|\int_{0}^{\infty} g(y) e^{-y^{2} / 2} \sin (x y) d y\right| e^{-x^{2} / 2} d x \\
& =\int_{0}^{\infty}\left|\int_{0}^{\infty} f(y) e^{-y^{2} / 2} \sin (x y) d y\right| e^{-x^{2} / 2} d x .
\end{aligned}
$$


Lemma 6.1. Define $I:[0, \infty) \rightarrow \mathbb{R}$ by

$$
I(y) \stackrel{\text { def }}{=} \int_{0}^{\infty} f(x) \exp \left(-\frac{x^{2}+y^{2}}{2}\right) \sin (x y) d x .
$$

Then I is $\sqrt{2}$-Lipschitz.

Proof. The Lipschitz condition follows from the following simple estimate on $\left|I^{\prime}\right|$ :

$$
\begin{aligned}
\left|I^{\prime}(y)\right| & \leqslant \int_{0}^{\infty} \exp \left(-\frac{x^{2}+y^{2}}{2}\right)|x \cos (x y)-y \sin (x y)| d x \\
& \leqslant \int_{0}^{\infty} \exp \left(-\frac{x^{2}+y^{2}}{2}\right) \sqrt{x^{2}+y^{2}} d x \\
& \leqslant \sqrt{2} e^{-y^{2} / 2} \int_{0}^{\infty} e^{-x^{2} / 2} \max \{x, y\} d x \\
& =\sqrt{2} e^{-y^{2} / 2}\left(\int_{0}^{y} e^{-x^{2} / 2} y d x+\int_{y}^{\infty} e^{-x^{2} / 2} x d x\right) \\
& \leqslant \sqrt{2} e^{-y^{2} / 2}\left(y^{2}+e^{-y^{2} / 2}\right) \leqslant \sqrt{2},
\end{aligned}
$$

where in the last inequality in (6.7) we used the bound $e^{-t}\left(2 t+e^{-t}\right) \leqslant 1$, which holds for all $t \geqslant 0$ since $e^{t}-e^{-t}=\sum_{k=0}^{\infty} 2 t^{2 k+1} /(2 k+1) ! \geqslant 2 t$.

LEMMA 6.2. For every $z \in[2 / 5,4 / 3]$, we have

$$
\int_{z-\frac{1}{4}}^{z+\frac{1}{4}} \int_{0}^{\infty} \exp \left(-\frac{x^{2}+y^{2}}{2}\right) \sin (x y) d x d y>\frac{3}{20} .
$$

Proof. Since for every $q \geqslant 0$ we have $\sin (q) \geqslant q-\frac{q^{3}}{6}+\frac{q^{5}}{120}-\frac{q^{7}}{5040}$,

$$
\begin{aligned}
& \int_{0}^{\infty} \exp \left(-\frac{x^{2}+y^{2}}{2}\right) \sin (x y) d x \\
& \geqslant \int_{0}^{\infty} \exp \left(-\frac{x^{2}+y^{2}}{2}\right)\left(x y-\frac{(x y)^{3}}{6}+\frac{(x y)^{5}}{120}-\frac{(x y)^{7}}{5040}\right) d x \\
& =-\frac{y e^{-y^{2} / 2}}{105}\left(y^{6}-7 y^{4}+35 y^{2}-105\right) \stackrel{\text { def }}{=} h(y) .
\end{aligned}
$$

We claim that there is a unique $w \in[0,2]$ at which $h^{\prime}$ vanishes, and moreover $h$ attains its maximum on $[0,2]$ at $w$. Indeed, $h^{\prime}(0)=e^{-y^{2} / 2}\left(y^{8}-14 y^{6}+70 y^{4}-\right.$ $\left.210 y^{2}+105\right) / 105$, and therefore $h^{\prime}(y)=1>0$ and $h^{\prime}(2)=-17 /\left(7 e^{2}\right)<0$, so it suffices to show that $h^{\prime}$ can have at most one zero on $[0,2]$. To this end, it 
suffices to show that the polynomial $p(y)=y^{8}-14 y^{6}+70 y^{4}-210 y^{2}+105$ is monotone on $[0,2]$. This is indeed the case, since for $y \in[0,2]$ we have $p^{\prime}(y)=-8 y^{5}\left(4-y^{2}\right)-y\left(52\left(y^{2}-35 / 13\right)^{2}+560 / 13\right)<0$.

The above reasoning shows that if we set $G(z)=\int_{z-1 / 4}^{z+1 / 4} h(y) d y$ then $G$ does not have local minima on [2/5,4/3]. Indeed, if $z \in[2 / 5,4 / 3]$ satisfies $G^{\prime}(z)=$ $h(z+1 / 4)-h(z-1 / 4)=0$ then $z-1 / 4<w<z+1 / 4$, implying that $h^{\prime}(z-1 / 4)>0$ and $h^{\prime}(z+1 / 4)<0$. Hence $G^{\prime \prime}(z)=h^{\prime}(z+1 / 4)-h^{\prime}(z-1 / 4)<0$, so $z$ cannot be a local minimum of $G$. Now, for every $z \in[2 / 5,4 / 3]$ we have

$$
\begin{aligned}
& \int_{z-\frac{1}{4}}^{z+\frac{1}{4}} \int_{0}^{\infty} \exp \left(-\frac{x^{2}+y^{2}}{2}\right) \sin (x y) d x d y \\
& \stackrel{(6.9)}{\geqslant} G(z) \geqslant \min \left\{G\left(\frac{2}{5}\right), G\left(\frac{4}{3}\right)\right\}>\frac{3}{20},
\end{aligned}
$$

where we used the fact that the above values of $G$ can be computed in closed form, for example $G(4 / 3)=(19047383 / 313528320) e^{-169 / 288}+$ $(131938921 / 313528320) e^{-361 / 288}>0.153$.

We will consider the following two quantities:

$$
M_{0} \stackrel{\text { def }}{=} \int_{0}^{\infty} \int_{0}^{\infty} \exp \left(-\frac{x^{2}+y^{2}}{2}\right) \sin (x y) d x d y=\frac{\log (1+\sqrt{2})}{\sqrt{2}}=0.6232 \ldots,
$$

and

$$
M_{1} \stackrel{\text { def }}{=} \int_{0}^{\infty} \int_{0}^{\infty} \exp \left(-\frac{x^{2}+y^{2}}{2}\right)|\sin (x y)| d x d y .
$$

Our goal is to show that $M=M_{0}$. Clearly, $M_{0} \leqslant M_{1}$. Our next (technical) lemma shows that $M_{1}$ is actually quite close to $M_{0}$.

LEMMA 6.3. $M_{1}-M_{0}<\frac{1}{20}$.

Proof. Since the integral in (6.11) converges very quickly and can therefore be computed numerically with high precision, Lemma 6.3 does not have much content. Nevertheless, we wish to explain how to reduce this lemma to an evaluation of an integral that can be computed in closed form. Let $p_{n}$ be the Taylor polynomial of degree $2 n-1$ of the function $x \mapsto \sqrt{1-x}$, that is,

$$
p_{n}(x)=\sum_{k=0}^{2 n-1}(-1)^{k}\left(\begin{array}{c}
1 / 2 \\
k
\end{array}\right) x^{k}=1+\sum_{k=1}^{2 n-1} \frac{\prod_{j=0}^{k-1}(2 j-1)}{2^{k} k !} x^{k} .
$$

Then $\sqrt{1-x} \leqslant p_{n}(x)$ for all $x \in(-1,1)$ and $n \in \mathbb{N}$. 
Now,

$$
\begin{aligned}
M_{1} & =\int_{0}^{\infty} \int_{0}^{\infty} \exp \left(-\frac{x^{2}+y^{2}}{2}\right) \sqrt{\frac{1-\cos (2 x y)}{2}} d x d y \\
& \leqslant \frac{1}{\sqrt{2}} \int_{0}^{\infty} \int_{0}^{\infty} \exp \left(-\frac{x^{2}+y^{2}}{2}\right) p_{n}(\cos (2 x y)) d x d y .
\end{aligned}
$$

For $j \in \mathbb{N}$, the integral $\int_{0}^{\infty} \int_{0}^{\infty} \exp \left(-\left(x^{2}+y^{2}\right) / 2\right)(\cos (2 x y))^{j} d x d y$ can be computed in closed form (it equals $\pi$ times a linear combination with rational coefficients of square roots of integers). One can therefore explicitly evaluate the integral on the right-hand side of (6.12) for $n=11$, obtaining the bound $M_{1}<0.671<M_{0}+0.05$.

LEMMA 6.4. $f$ and $g$ are constant on the interval [2/5, 4/3].

Proof. Assume for contradiction that $g$ is not constant on [2/5, 4/3]. By (6.4), we know that $g=\operatorname{sign}(I)$, where $I$ is given in (6.6). Hence there is some $z \in[2 / 5,4 / 3]$ such that $I(z)=0$. By Lemma 6.1, we therefore know that $|I(y)| \leqslant \sqrt{2}|y-z|$ for all $y \in[0, \infty)$. Hence,

$$
\begin{aligned}
M_{0} \leqslant & M \stackrel{(6.5)}{\leqslant} \int_{[0, z-1 / 4] \cup[z+1 / 4, \infty)} \int_{0}^{\infty} \exp \left(-\frac{x^{2}+y^{2}}{2}\right)|\sin (x y)| d x d y \\
& +\int_{z-\frac{1}{4}}^{z+\frac{1}{4}}|I(y)| d y \\
& \leqslant M_{1}-\int_{z-\frac{1}{4}}^{z+\frac{1}{4}} \int_{0}^{\infty} \exp \left(-\frac{x^{2}+y^{2}}{2}\right)|\sin (x y)| d x d y+\frac{\sqrt{2}}{16} \\
\stackrel{(6.8)}{<} & M_{1}-\frac{3}{20}+\frac{\sqrt{2}}{16}<M_{1}-\frac{3}{50} .
\end{aligned}
$$

This contradicts Lemma 6.3.

Before proceeding to the proof of Theorem 1.4, we record one more elementary lemma.

LEMMA 6.5. For every $a>0$, we have

$$
\int_{a}^{\infty} e^{-x^{2} / 2} d x<\frac{16}{3 e^{2} a^{3}} .
$$

Proof. Set $c=16 /\left(3 e^{2}\right)$ and $\psi(a)=\left(c / a^{3}\right)-\int_{a}^{\infty} e^{-x^{2} / 2} d x$. Since $\lim _{a \rightarrow \infty}$ $\psi(a)=0$, it suffices to show that $\psi$ is decreasing on $(0, \infty)$. Because $\psi^{\prime}(a)=$ $e^{-a^{2} / 2}-3 c / a^{4}$, our goal is to show that $e^{a^{2} / 2} \geqslant a^{4} /(3 c)$, or equivalently 
that $a^{2} \geqslant 8 \log a-2 \log (3 c)$. Set $\rho(a)=a^{2}-8 \log a+2 \log (3 c)$. Since $\rho^{\prime}(a)=$ $2 a-8 / a$, the minimum of $\rho$ is attained at $a=2$. We are done, since by the choice of $c$ we have $\rho(2)=0$.

Proof of Theorem 1.4. Due to Lemma 6.4, we may assume from now on that $f(x)=1$ for all $x \in[2 / 5,4 / 3]$. Our goal is to show that, under this assumption, $f=g=1$. We shall achieve this in a few steps.

We will first show that $f(y)=g(y)=1$ for all $y \in[0,4 / 3]$. To see this, fix $y \in(0,1 / 2]$, and note that the function $x \mapsto \sin (x y)$ is concave on $[0,4 / 3]$ (since in this range we have $x y \leqslant 2 / 3 \leqslant \pi / 2$ ). It follows that for $x \in[0,4 / 3]$ we have $\sin (x y) \geqslant \sin (4 y / 3) /(4 / 3) x$, and for $x \in[4 / 3, \infty)$ we have $|\sin (x y)| \leqslant$ $\sin (4 y / 3) /(4 / 3) x$. Using this fact, the fact that $|\sin (x y)| \leqslant x y$ for all $x \geqslant 0$, and the fact that $f=1$ on $[2 / 5,4 / 3]$, we deduce that

$$
\begin{aligned}
\int_{0}^{\infty} e^{-x^{2} / 2} \sin (x y) f(x) d x \geqslant & -\int_{0}^{\frac{2}{5}} e^{-x^{2} / 2} x y d x+\int_{\frac{2}{5}}^{\frac{4}{3}} e^{-x^{2} / 2} \frac{\sin (4 y / 3)}{4 / 3} x d x \\
& -\int_{\frac{4}{3}}^{\infty} e^{-x^{2} / 2} \frac{\sin (4 y / 3)}{4 / 3} x d x \\
= & -\left(1-e^{-2 / 25}\right) y+\frac{\sin (4 y / 3)}{4 / 3}\left(e^{-2 / 25}-2 e^{-8 / 9}\right) \\
& \stackrel{\text { def }}{=} r(y) .
\end{aligned}
$$

Note that for $z \in[0,1 / 2]$ we have

$$
\begin{aligned}
r^{\prime}(z) & =\cos \left(\frac{4 z}{3}\right)\left(e^{-2 / 25}-2 e^{-8 / 9}\right)-\left(1-e^{-2 / 25}\right) \\
& \geqslant \cos \left(\frac{2}{3}\right)\left(e^{-2 / 25}-2 e^{-8 / 9}\right)-\left(1-e^{-2 / 25}\right)>0.002>0 .
\end{aligned}
$$

Hence $r$ is increasing on [0,1/2], and in particular $r(y)>r(0)=0$. By (6.4) and (6.14), it follows that $g(y)=1$ for all $y \in[0,1 / 2]$. Since Lemma 6.4 tells us that $g$ is constant on $[2 / 5,4 / 3]$, it follows that $g=1$ on $[0,4 / 3]$. We my now repeat the above argument with the roles of $f$ and $g$ interchanged, deducing that $f=g=1$ on $[0,4 / 3]$.

Our next goal is to show that $f=g=1$ on $[0,3 \pi / 4]$. We already know that $f=g=1$ on $[0,4 / 3]$, so assume that $y \in[4 / 3,3 \pi / 4]$. Then $4 y / 3 \geqslant(4 / 3)^{2}>$ $\pi / 2$ and $4 y / 3 \leqslant \pi$, implying that

$$
\int_{0}^{4 / 3} f(x) e^{-x^{2} / 2} \sin (x y) d x \geqslant \int_{0}^{\pi / 2 y} e^{-x^{2} / 2} \sin (x y) d x \geqslant \int_{0}^{*} e^{-x^{2} / 2} \frac{2 x y}{\pi} d x
$$




$$
\begin{aligned}
& =\frac{2 y}{\pi}\left(1-e^{-\pi^{2} /\left(8 y^{2}\right)}\right) \stackrel{(* *)}{\geqslant} \frac{2 y}{\pi}\left(\frac{\pi^{2}}{8 y^{2}}-\frac{\pi^{4}}{128 y^{4}}\right) \\
& =\frac{\pi}{4 y}-\frac{\pi^{3}}{64 y^{3}} \stackrel{(* * *)}{\geqslant} \frac{8}{27},
\end{aligned}
$$

where in $(*)$ we used the fact that $\sin (z) \geqslant 2 z / \pi$ for $z \in[0, \pi / 2]$, in $(* *)$ we used the elementary inequality $1-e^{-z} \geqslant z-z^{2} / 2$, which holds for all $z \geqslant 0$, and in $(* * *)$ we used the fact that the function $y \mapsto \pi / 4 y-\pi^{3} / 64 y^{3}$ has a unique local maximum on $(1, \infty)$, which implies that its minimum on $[4 / 3,3 \pi / 4]$ is attained at the endpoints, and therefore its minimum on [4/3,3 $3 / 4]$ equals $8 / 27$.

Now,

$$
\begin{aligned}
\int_{0}^{\infty} f(x) e^{-x^{2} / 2} \sin (x y) d x & \geqslant \frac{(6.15)}{27}-\int_{\frac{4}{3}}^{\infty} e^{-x^{2} / 2} d x \\
& =\frac{8}{27}-\left(\sqrt{\frac{\pi}{2}}-\int_{0}^{\frac{4}{3}} e^{-x^{2} / 2} d x\right) \\
& \geqslant \frac{8}{27}-\sqrt{\frac{\pi}{2}}+\int_{0}^{\frac{4}{3}}\left(1-\frac{x^{2}}{2}+\frac{x^{4}}{8}-\frac{x^{6}}{48}\right) d x \\
& =\frac{302572}{229635}-\sqrt{\frac{\pi}{2}}>0 .
\end{aligned}
$$

Using (6.4), we deduce from (6.16) that $g(y)=1$. By symmetry, the same argument applies to $f$, implying that $f=g=1$ on $[0,3 \pi / 4]$.

Let $A \geqslant 3 \pi / 4$ be the supremum over those $a>0$ such that $f=g=1$ on $[0, a]$. Our goal is to show that $A=\infty$, so assume for contradiction that $A$ is finite.

Note that, for every $y>0$ and every $k \in \mathbb{N} \cup\{0\}$,

$$
\begin{aligned}
\int_{2 k \pi / y}^{2(k+1) \pi / y} e^{-x^{2} / 2} \sin (x y) d x & =\int_{2 k \pi / y}^{(2 k+1) \pi / y}\left(e^{-x^{2} / 2}-e^{-(x+\pi / y)^{2} / 2}\right) \sin (x y) d x \\
& \geqslant 0 .
\end{aligned}
$$

It follows that if $A \in[2 k \pi / y, 2(k+1) \pi / y]$ then

$$
\int_{2 k \pi / y}^{A} e^{-x^{2} / 2} \sin (x y) d x \geqslant 0 .
$$

To check (6.18), note that for $A \in[2 k \pi / y,(2 k+1) \pi / y]$ the integrand in (6.18) is nonnegative, and for $A \in[(2 k+1) \pi / y, 2(k+1) \pi / y]$ the integral in (6.18) is at least the integral on the left-hand side of (6.17). Assume from now on that $y>A$, and note that since $A \geqslant 3 \pi / 4$ we have $3 \pi /(2 y)<A$. This implies the following 
bound:

$$
\begin{aligned}
\int_{0}^{\min \{2 \pi / y, A\}} e^{-x^{2} / 2} \sin (x y) d x & \geqslant \int_{0}^{2 \pi / y} e^{-x^{2} / 2} \sin (x y) d x \\
& =\int_{0}^{\pi / y}\left(e^{-x^{2} / 2}-e^{-(x+\pi / y)^{2} / 2}\right) \sin (x y) d x \\
& =\int_{0}^{\pi / y} e^{-x^{2} / 2}\left(1-e^{-\pi x / y} e^{-(\pi / y)^{2} / 2}\right) \sin (x y) d x \\
& \geqslant\left(1-e^{-(\pi / y)^{2} / 2}\right) \int_{0}^{\pi / 2 y} e^{-x^{2} / 2} \frac{2 x y}{\pi} d x \\
& =\frac{2 y}{\pi}\left(1-e^{-\pi^{2} /\left(2 y^{2}\right)}\right)\left(1-e^{-\pi^{2} /\left(8 y^{2}\right)}\right) \\
& \geqslant \frac{2 y}{\pi}\left(\frac{\pi^{2}}{2 y^{2}}-\frac{\pi^{4}}{8 y^{4}}\right)\left(\frac{\pi^{2}}{8 y^{2}}-\frac{\pi^{4}}{128 y^{4}}\right) \\
& \geqslant \frac{5 \pi^{3}}{81 y^{3}},
\end{aligned}
$$

where in the last inequality of (6.19) we used the fact that $y>A \geqslant 3 \pi / 4$.

A combination of (6.17), (6.18), and (6.19) with the fact that $f=1$ on $[0, A)$ shows that

$$
\int_{0}^{A} f(x) e^{-x^{2} / 2} \sin (x y) d x \geqslant \frac{5 \pi^{3}}{81 y^{3}},
$$

and therefore, by Lemma 6.5,

$$
\int_{0}^{\infty} f(x) e^{-x^{2} / 2} \sin (x y) d x \geqslant \frac{5 \pi^{3}}{81 y^{3}}-\int_{A}^{\infty} e^{-x^{2} / 2} d x \stackrel{(6.13)}{\geqslant} \frac{5 \pi^{3}}{81 y^{3}}-\frac{16}{3 e^{2} A^{3}} .
$$

The right-hand side of (6.20) is positive provided that $y \leqslant 5 A / 4$. By (6.4), this means that $g=1$ on $[A, 5 A / 4]$, and hence also on [0,5A/4]. By symmetry, $f=1$ on $[0,5 A / 4]$ as well, contradicting the definition of $A$.

\section{Acknowledgements}

M. B. was supported in part by an NSERC Discovery Grant. A. N. was supported in part by NSF grant CCF-0832795, BSF grant 2006009, and the Packard Foundation. Part of this work was completed when A. N. was participating in the Discrete Analysis program at the Isaac Newton Institute for Mathematical Sciences. An extended abstract describing the contents of this work appeared in the 52nd Annual IEEE Symposium on Foundations of Computer Science. 


\section{References}

[1] F. Albiac and N. J. Kalton, Topics in Banach Space Theory, Graduate Texts in Mathematics, 233 (Springer, New York, 2006).

[2] N. Alon and A. Naor, 'Approximating the cut-norm via Grothendieck's inequality', SIAM J. Comput. 35(4) (2006), 787-803 (electronic).

[3] G. E. Andrews, R. Askey and R. Roy, Special Functions, Encyclopedia of Mathematics and its Applications, 71 (Cambridge University Press, Cambridge, 1999).

[4] R. Azor, J. Gillis and J. D. Victor, 'Combinatorial applications of Hermite polynomials', SIAM J. Math. Anal. 13(5) (1982), 879-890.

[5] R. Blei, Analysis in Integer and Fractional Dimensions, Cambridge Studies in Advanced Mathematics, 71 (Cambridge University Press, Cambridge, 2001).

[6] R. C. Blei, 'An elementary proof of the Grothendieck inequality', Proc. Amer. Math. Soc. 100(1) (1987), 58-60.

[7] R. Cleve, P. Høyer, B. Toner and J. Watrous, 'Consequences and limits of nonlocal strategies', 19th Annual IEEE Conference on Computational Complexity (2004), 236-249.

[8] A. M. Davie, 'Matrix norms related to Grothendieck's inequality', in Banach Spaces (Columbia, Mo., 1984), Lecture Notes in Mathematics, 1166 (Springer, Berlin, 1985), 22-26.

[9] J. Diestel, J. H. Fourie and J. Swart, The Metric Theory of Tensor Products (American Mathematical Society, Providence, RI, 2008), Grothendieck's résumé revisited.

[10] J. Diestel, H. Jarchow and A. Tonge, Absolutely Summing Operators, Cambridge Studies in Advanced Mathematics, 43 (Cambridge University Press, Cambridge, 1995).

[11] P. C. Fishburn and J. A. Reeds, 'Bell inequalities, Grothendieck's constant, and root two', SIAM J. Discrete Math. 7(1) (1994), 48-56.

[12] A. Frieze and R. Kannan, 'Quick approximation to matrices and applications', Combinatorica 19(2) (1999), 175-220.

[13] D. J. H. Garling, Inequalities: A Journey into Linear Analysis (Cambridge University Press, Cambridge, 2007).

[14] A. Grothendieck, 'Résumé de la théorie métrique des produits tensoriels topologiques', Bol. Soc. Mat. São Paulo 8 (1953), 1-79.

[15] M. Grötschel, L. Lovász and A. Schrijver, 'The ellipsoid method and its consequences in combinatorial optimization', Combinatorica 1(2) (1981), 169-197.

[16] U. Haagerup, 'A new upper bound for the complex Grothendieck constant', Israel J. Math. 60(2) (1987), 199-224.

[17] G. J. O. Jameson, Summing and Nuclear Norms in Banach Space Theory, London Mathematical Society Student Texts, 8 (Cambridge University Press, Cambridge, 1987).

[18] W. B. Johnson and J. Lindenstrauss, 'Basic concepts in the geometry of Banach spaces', in Handbook of the Geometry of Banach Spaces, Vol. I (North-Holland, Amsterdam, 2001), $1-84$.

[19] S. Khot, 'On the power of unique 2-prover 1-round games', in Proceedings of the Thirty-Fourth Annual ACM Symposium on Theory of Computing (ACM, New York, 2002), 767-775 (electronic).

[20] S. Khot, G. Kindler, E. Mossel and R. O’Donnell, 'Optimal inapproximability results for MAX-CUT and other 2-variable CSPs?', SIAM J. Comput. 37(1) (2007), 319-357 (electronic).

[21] S. Khot and A. Naor, 'Grothendieck-type inequalities in combinatorial optimization', Comm. Pure Appl. Math. 65(7) (2012), 992-1035. 
[22] H. König, 'On an extremal problem originating in questions of unconditional convergence', in Recent Progress in Multivariate Approximation (Witten-Bommerholz, 2000), Internat. Ser. Numer. Math., 137 (Birkhäuser, Basel, 2001), 185-192.

[23] J.-L. Krivine, 'Sur la constante de Grothendieck', C. R. Acad. Sci. Paris Sér. A-B 284(8) (1977), A445-A446.

[24] J.-L. Krivine, 'Constantes de Grothendieck et fonctions de type positif sur les sphères', Adv. Math. 31(1) (1979), 16-30.

[25] E. H. Lieb, 'Gaussian kernels have only Gaussian maximizers', Invent. Math. 102(1) (1990), 179-208.

[26] J. Lindenstrauss and A. Pełczyński, 'Absolutely summing operators in $L_{p}$-spaces and their applications', Studia Math. 29 (1968), 275-326.

[27] J. Lindenstrauss and L. Tzafriri, Classical Banach Spaces. I. Sequence Spaces, Ergebnisse der Mathematik und ihrer Grenzgebiete, 92 (Springer, Berlin, 1977).

[28] B. Maurey, 'Une nouvelle démonstration d'un théorème de Grothendieck', in Séminaire Maurey-Schwartz Année 1972-1973: Espaces $L^{p}$ et applications radonifiantes, Exp. No. 22 (Centre de Math., École Polytech, Paris, 1973), 7.

[29] B. Maurey and G. Pisier, 'Un théorème d'extrapolation et ses conséquences', C. R. Acad. Sci. Paris Sér. A-B 277 (1973), A39-A42.

[30] A. Naor and O. Regev, 'Krivine schemes are optimal', Proc. Amer. Math. Soc., 2012, to appear, preprint available at http://arxiv.org/abs/1205.6415.

[31] G. Pisier, 'Grothendieck's theorem for noncommutative $C^{*}$-algebras, with an appendix on Grothendieck's constants', J. Funct. Anal. 29(3) (1978), 397-415.

[32] G. Pisier, Factorization of Linear Operators and Geometry of Banach Spaces, CBMS Regional Conference Series in Mathematics, 60 (Published for the Conference Board of the Mathematical Sciences, Washington, DC, 1986).

[33] G. Pisier, 'Grothendieck's theorem, past and present', Bull. Amer. Math. Soc. (N.S.) 49(2) (2012), 237-323.

[34] P. Raghavendra and D. Steurer, 'Towards computing the Grothendieck constant', Proceedings of the Twentieth Annual ACM-SIAM Symposium on Discrete Algorithms, 2009, $525-534$.

[35] J. A. Reeds, 'A new lower bound on the real Grothendieck constant', unpublished manuscript, available at http://www.dtc.umn.edu/reedsj/bound2.dvi 1991.

[36] R. E. Rietz, 'A proof of the Grothendieck inequality', Israel J. Math. 19 (1974), 271-276.

[37] L. Schwartz, Geometry and Probability in Banach Spaces, Lecture Notes in Mathematics, 852 (Springer, Berlin, 1981), based on notes taken by Paul R. Chernoff.

[38] B. S. Tsirelson, 'Quantum analogues of Bell's inequalities. The case of two spatially divided domains', Zap. Nauchn. Sem. Leningrad. Otdel. Mat. Inst. Steklov. (LOMI) 142 (1985), 174-194, 200. Problems of the theory of probability distributions, IX. 